\title{
Limits of the Improved Integrability of the Volume Forms
}

\author{
Luigi Greco, Tadeusz Iwaniec $\mathcal{G}$ Gioconda Moscariello
}

ABSTRACT. We identify the exact degree of integrability of nonnegative volume forms and the Jacobians of orientation preserving mappings from various Orlicz-Sobolev classes. An improvement takes place when the Jacobian belongs to the Orlicz space $L^{\Psi}(\Omega)$, where $\Psi$ grows almost linearly, that is, $t^{1-\varepsilon} \prec \Psi(t) \prec t^{1+\varepsilon}$ for $\varepsilon>0$. Our results amount to the principle: the further the Jacobian is from $L_{\mathrm{loc}}^{1}(\Omega)$, the less is the improvement of integrability. In fact, as shown in [LZ], [Wu], [GIM], the largest improvement happens when the Jacobian is precisely in the space $L_{\text {loc }}^{1}(\Omega)$.

\section{Contents.}

1. Introduction . . . . . . . . . . . . . . . . 305

2. Notation . . . . . . . . . . . . . . . . . . 307

3. Main results . . . . . . . . . . . . . . . . . . . 311

4. Hölder inequality . . . . . . . . . . . . . . . . . . 316

5. Maximal inequalities in generalized Orlicz spaces . . . . . 317

6. Proof of Theorem $1 \ldots \ldots \ldots$. . . . . . . . . . . 321

7. An example . . . . . . . . . . . . . . . . . . . . 323

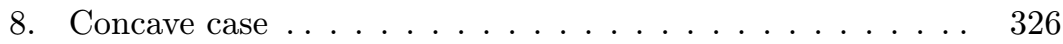

9. Some conclusions . . . . . . . . . . . . . . . . 331

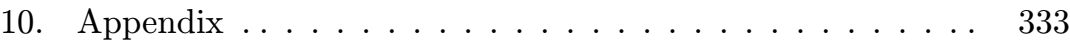

11. References .................... 338

1. Introduction. Let $\Omega$ be an open subset of $\mathbb{R}^{n}$ and let $f=\left(f^{1}, \ldots, f^{n}\right)$ : $\Omega \rightarrow \mathbb{R}^{n}$ be a mapping whose distributional differential $D f: \Omega \rightarrow G L(n)$ is a locally integrable function on $\Omega$ with values in the space $G L(n)$ of all $n \times n$ matrices. In particular, its Jacobian determinant,

$$
J=J(x, f)=\operatorname{det} D f(x)=\operatorname{det}\left(\frac{\partial f^{i}}{\partial x_{j}}\right),
$$


is defined point-wise at almost every $x \in \Omega$. Using notation of exterior algebra, we can write

$$
J(x, f) d x=d f^{1} \wedge \cdots \wedge d f^{n} .
$$

Thus $f$ induces a volume form on $\mathbb{R}^{n}$. Our basic assumption is that $J(x, f) \geq 0$ a.e., that is, $f$ is an orientation preserving mapping.

In recent years, the Jacobian function has been subjected to a great deal of investigation. Its rather special properties were already recognized in the late 1960's when F. W. Gehring, J. Väisälä and Y. Reshetnyak initiated an extensive study of the geometric mapping theory. It seems appropriate here to recall the celebrated paper of F. W. Gehring [G] in which he invented reverse Hölder inequalities. He used these inequalities to establish the $L^{1+\varepsilon}$-integrability of the Jacobian of a quasiconformal mapping. Reshetnyak's result on weak continuity of the Jacobian function is also particularly remarkable $[R]$.

In more recent years, there has been intensive study of nonlinear elasticity theory, in which nonlinear quantities (null Lagrangians), somewhat resembling Jacobians, play an important role. However, no attempt has been made to develop systematic tools for studying such quantities until Stefan Müller [M] discovered that $J \log J \in L_{\text {loc }}^{1}(\Omega)$ if $D f \in L^{n}(\Omega)$. A careful examination of Müller's approach leads to local estimates of the form

$$
\int_{\sigma Q} J(x, f) \log \left(e+\frac{J(x, f)}{J_{\sigma Q}}\right) d x \leq \frac{C(n)}{\sigma^{n}(1-\sigma)} \int_{Q}|D f(x)|^{n} d x
$$

for every subcube $Q \subset \Omega$ with constant independent of $Q$. Here and subsequently, $\sigma Q$ stands for the cube with the same center as $Q$, but $\sigma$ times as small, $0<\sigma<1$, and $J_{\sigma Q}$ is the integral mean of $J$ over the cube $\sigma Q$.

Because of its compelling beauty and geometric significance, the subject has been greatly expanded, engaging the attention of many researchers [IS1], [IL], [CLMS], [T], [BFS], [DMa], [DMu], [Do], [Mo], [Mi2], [GIM], [LZ], [LMZ], [LMWZ], [Wu], [Z], [Gr1], [Gr2], [GI]. The latter illustrates how the local estimates (1.3) can be used to obtain even higher integrability of the Jacobian. Müller's proof actually shows that the maximal function of $J$ is locally integrable. However, this method has the disadvantage of requiring that the Jacobian be a priori integrable. In order to get something interesting for mappings with nonintegrable Jacobian, it was necessary to develop new technique. The idea goes back at least as far as [I1]. Subsequently, a result dual to that of Müller has been obtained in [IS1]. It states that the Jacobian is locally integrable if $|D f|$ belongs to the Zygmund class $L^{n} \log ^{-1} L$, that is, $|D f|^{n} \log (e+|D f|) \in L^{1}(\Omega)$. More precisely, we have

$$
f_{\sigma Q} J(x, f) d x \leq \frac{c(n)}{\sigma^{n}(1-\sigma)}\left(f_{Q}|D f(x)|^{n^{2} /(n+1)} d x\right)^{(n+1) / n},
$$


for each subcube $Q \subset \Omega$ and $0<\sigma<1$. Here the integrals are given a bar to indicate their mean values, that is $f_{Q}=\left(\int_{Q}\right) /|Q|$, where $|Q|$ denotes the volume of $Q$.

By far the most difficult was to relax the condition $|D f| \in L^{n}(\Omega)$ - the natural space in which to consider the Jacobian. Critical to this result were estimates "below the natural exponent" for certain non-linear PDE's, [IM], [I1], [IS2], [IL]. A remarkable connection with Rochberg-Weiss theory of non-linear commutators and interpolation $[\mathrm{RW}]$ has been found in the course of these developments. But we will touch only a few aspects of this subject. For more details, we refer to the very recent work by Mario Milman [Mi1], [Mi2], [Mi3]. A comprehensive account of $L^{p}$-integrability properties of null Lagrangians has been made in [IL], which proves rather clearly that differential forms are fundamental tools for dealing with the Jacobians. For more developments see [I3].

The aim of this paper is to bring together all of the above ideas and look more closely at the phenomenon of the improved integrability of the Jacobian and somewhat more general expressions, called volume forms. To formulate the results we must first set up some notation and terminology.

2. Notation. In this section we summarize without proof the relevant material on exterior algebra, Orlicz spaces and differential forms. A more complete notation and some theory is given in the subsequent sections.

Exterior algebra. We will denote by $\Lambda^{\ell}=\Lambda^{\ell}\left(\mathbb{R}^{n}\right)$ the linear space of the $\ell$-covectors on $\mathbb{R}^{n}, \ell=1,2, \ldots, n$, also called exterior forms of degree $\ell$. It is a normed space of dimension $\left(\begin{array}{l}n \\ \ell\end{array}\right)$. As a rule, bold letters will be used to denote finite tuples. For example, we shall consider an $m$-tuple $\boldsymbol{\ell}=\left(\ell_{1}, \ldots, \ell_{m}\right)$ of positive integers such that $\ell_{1}+\cdots+\ell_{m}=n$. The symbol $\Lambda^{\ell}=\Lambda^{\ell_{1}} \times \cdots \times \Lambda^{\ell_{m}}$ stands for the set of $m$-tuples $\boldsymbol{\omega}=\left(\omega_{1}, \ldots, \omega_{m}\right)$ of covectors $\omega_{i} \in \Lambda^{\ell_{i}}\left(\mathbb{R}^{n}\right)$, $i=1,2, \ldots, m$. To each such $\boldsymbol{\omega}$ there corresponds the $n$-covector (volume element) $\omega=\omega_{1} \wedge \cdots \wedge \omega_{m} \in \Lambda^{n}\left(\mathbb{R}^{n}\right)$, sometimes denoted by $\operatorname{det} \boldsymbol{\omega}$ to indicate a connection with determinants. With this notation, Hadamard's inequality writes as

$$
|\operatorname{det} \boldsymbol{\omega}| \leq C(\ell)\left|\omega_{1}\right| \cdots\left|\omega_{m}\right| .
$$

It is of independent interest to identify the smallest constant $C(\ell)$ and the extremal case in this inequality.

From here on, we will fix the standard ordered basis for $\mathbb{R}^{n}$, so it makes sense to speak of non-negative volume elements. 
Orlicz spaces. Here $\Omega$ stands for a cube in $\mathbb{R}^{n}$. Functions which are equals a.e. are identified. A continuously increasing function on $\mathbb{R}_{+}=[0, \infty]$, say $\Psi: \mathbb{R}_{+} \rightarrow \mathbb{R}_{+}$, such that $\Psi(0)=0$ and $\Psi(\infty)=\infty$, will be referred to as an Orlicz function. The generalized Orlicz space, denoted by $\mathcal{L}^{\Psi}(\Omega)=\mathcal{L}^{\Psi}(\Omega, X)$, consists of all functions $g: \Omega \rightarrow X$ with values in a finite dimensional normed space $(X,|\cdot|)$ such that

$$
\int_{\Omega} \Psi\left(k^{-1}|g|\right)<\infty
$$

for some $k=k(g)>0$. It is a complete linear metric space $[\mathrm{RR}]$. For simplicity of notation, we ignore the dependence of $\mathcal{L}^{\Psi}(\Omega)$ on $X$ when no confusion can arise. There is a non-linear functional on $\mathcal{L}^{\Psi}(\Omega)$ defined by

$$
\|g\|_{\Psi}=\|g\|_{\Psi, \Omega}=\inf \left\{k>0 ; f_{\Omega} \Psi\left(k^{-1}|g|\right) \leq \Psi(1)\right\}
$$

where $f_{\Omega}$ stands for the integral mean over $\Omega$. For abbreviation, we write $\|g\|_{\Psi}$ instead of $\|g\|_{\Psi, \Omega}$ when $\Omega$ is fixed. If, moreover, $\Psi$ is convex then \|\|$_{\Psi}$ is the familiar Luxemburg norm, which makes $\mathcal{L}^{\Psi}(\Omega)$ a Banach space. However, our basic Orlicz functions will be only log-convex. A function $\Phi: \mathbb{R}_{+} \rightarrow \mathbb{R}_{+}$, $\Phi(0)=0$, is said to be log-convex if $t \mapsto t \Phi^{\prime}(t)$ is increasing on $[0, \infty)$. In addition, we shall require that $\Phi$ is normalized by $\Phi^{\prime}(1)=1$. Every $m$-tuple $\boldsymbol{\Phi}=\left(\Phi_{1}, \ldots, \Phi_{m}\right)$ of log-convex functions gives rise to a log-convex function $\Phi$, which is uniquely determined from the formula

$$
\left(t \Phi^{\prime}\right)^{-1}=\left(t \Phi_{1}^{\prime}\right)^{-1} \cdots\left(t \Phi_{m}^{\prime}\right)^{-1} .
$$

Hereafter, the symbol $\left(t \Phi^{\prime}\right)^{-1}$ stands for the inverse function of $t \Phi^{\prime}(t)$. Because of Hölder's inequality, see Section 4 , we call $\Phi$ the Hölder product of $\Phi_{1}, \ldots, \Phi_{m}$, the latter being called Hölder factors of $\Phi$. We have

$$
\left\|g_{1} \cdots g_{m}\right\|_{\Phi} \leq\left\|g_{1}\right\|_{\Phi_{1}} \cdots\left\|g_{m}\right\|_{\Phi_{m}}
$$

for all $g_{i} \in \mathcal{L}^{\Phi_{i}}(\Omega), i=1,2, \ldots, m$. In case $\Phi(t)=t$, we say that $\Phi_{1}, \ldots, \Phi_{m}$ are Hölder conjugate. Of course, Hölder's multiplication is an associative operation, and every log-convex function $\Phi$ can be factored in many ways. One useful factorization of $\Phi$ is obtained by setting

$$
\Phi_{i}(t)=\frac{1}{p_{i}} \Phi\left(t^{p_{i}}\right), \quad i=1,2, \ldots, m
$$

where $1<p_{1}, \ldots, p_{m}<\infty$ are Hölder conjugate numbers, that is, $1 / p_{1}+\cdots+$ $1 / p_{m}=1$. Then we have $\|g\|_{\Phi_{i}}^{p_{i}}=\left\||g|^{p_{i}}\right\|_{\Phi}$, for $g \in \mathcal{L}^{\Phi_{i}}(\Omega)$. For many purposes, 
however, it is immaterial which factors $\Phi_{1}, \ldots, \Phi_{m}$ are chosen to define $\Phi$ as long as $\Phi$ is log-convex and satisfies certain regularity conditions.

Although we do not require $\Delta_{2}$-regularity of the Orlicz functions in any essential way, it is appropriate to recall this notion. Thus $\Phi: \mathbb{R}_{+} \rightarrow \mathbb{R}_{+}$is $\Delta_{2}$-regular if $\Phi(2 t) \leq K \Phi(t)$ for some $K>1$ and all $t \geq 0$.

Most of the estimates presented here remain valid if the Orlicz functions are replaced by equivalent ones. It is said that $\Psi$ is stronger than $\Phi, \Psi \succeq \Phi$ for short, if for every $\varepsilon>0$ there exists $K=K(\varepsilon)>0$ such that

$$
\Phi(t) \leq \varepsilon \Psi(K t) \quad \text { for all } t \geq 0 .
$$

If $\Psi \succeq \Phi \succeq \Psi$, we say that $\Phi$ and $\Psi$ are equivalent and we abbreviate it to

$$
\Phi \sim \Psi .
$$

We write $\Psi \succ \Phi$ if $\Psi$ is stronger than $\Phi$ and not equivalent to $\Phi$. We leave it to the reader to verify that the condition $\Phi \preceq \Psi$ implies $\mathcal{L}^{\Psi}(\Omega) \subset \mathcal{L}^{\Phi}(\Omega)$ and

$$
\|g\|_{\Phi} \leq C\|g\|_{\Psi}
$$

where $C=K(\Phi(1) / \Psi(1))$.

Differential Forms. Although the notion of a differential form is usually placed in the context of a manifold, we confine ourselves to a cube $\Omega \subset \mathbb{R}^{n}$ as the base domain. In this case a differential form of degree $\ell$ is simply a function on $\Omega$ with values in $\Lambda^{\ell}\left(\mathbb{R}^{n}\right)$. Accordingly, for a given Orlicz function $\Phi: \mathbb{R}_{+} \rightarrow \mathbb{R}_{+}$, the $\Phi$-space of differential $\ell$-forms is denoted by $\mathcal{L}^{\Phi}\left(\Omega, \Lambda^{\ell}\right)$. An element $\alpha \in \mathcal{L}^{\Phi}\left(\Omega, \Lambda^{\ell}\right)$ is said to be closed if it is locally integrable and $d \alpha=0$ in the sense of distributions, where $d$ denotes the exterior differentiation. Let us point out in advance that when referring to a closed form $\alpha \in \mathcal{L}^{\Phi}\left(\Omega, \Lambda^{\ell}\right)$, we shall always have $\Phi(t) \succeq t$ which ensures that $\alpha$ is indeed locally integrable.

Note that the wedge product $\omega_{1} \wedge \cdots \wedge \omega_{m}$ of closed differential forms $\omega_{i} \in$ $\mathcal{L}^{\Phi_{i}}\left(\Omega, \Lambda^{\ell_{i}}\right)$ need not be a closed form, even if we assume that $\omega_{1} \wedge \cdots \wedge \omega_{m}$ is locally integrable. For this to happen, it suffices that $\Phi_{1}, \ldots, \Phi_{m}$ are convex and $\Delta_{2}$-regular, with Hölder product $\Phi(t) \succeq t$. This remark is particularly important when applying our results to differential forms $\omega^{I}=d f^{i_{1}} \wedge \cdots \wedge d f^{i_{\ell}}$, where $f^{i_{1}}, \ldots, f^{i_{\ell}}$ are coordinate functions of a mapping $f=\left(f^{1}, \ldots, f^{n}\right): \Omega \rightarrow$ $\mathbb{R}^{n}$ of a certain Sobolev class.

Let $\boldsymbol{\ell}=\left(\ell_{1}, \ldots, \ell_{m}\right)$ be an $m$-tuple of positive integers with $\ell_{1}+\cdots+\ell_{m}=$ $n$ and let $\boldsymbol{\Phi}=\left(\Phi_{1}, \ldots, \Phi_{m}\right)$ be an $m$-tuple of log-convex functions such that $\Phi(t) \succeq t$ for $i=1,2, \ldots, m$. We consider mappings $\boldsymbol{\omega}=\left(\omega_{1}, \ldots, \omega_{m}\right): \Omega \rightarrow \Lambda^{\ell}=$ $\Lambda^{\ell_{1}} \times \cdots \times \Lambda^{\ell_{m}}$ where $\omega_{i} \in \mathcal{L}^{\Phi_{i}}\left(\Omega, \Lambda^{\ell_{i}}\right), i=1,2, \ldots, m$, are closed forms whose wedge product $\omega=\operatorname{det} \boldsymbol{\omega}=\omega_{1} \wedge \cdots \wedge \omega_{m}$ is non-negative (volume form). For use 
with upcoming results, we denote the class of all such mappings by $\mathcal{A}^{\Phi}\left(\Omega, \Lambda^{\ell}\right)$. Hadamard's inequality yields

$$
\|\operatorname{det} \boldsymbol{\omega}\|_{\Phi} \leq C(\ell)\left\|\omega_{1}\right\|_{\Phi_{1}} \cdots\left\|\omega_{m}\right\|_{\Phi_{m}} .
$$

Here we recall that $\Phi$ stands for the Hölder product of $\Phi_{1}, \ldots, \Phi_{m}$. The Orlicz function $\Phi$ will be of more interest than its factors. Therefore, abusing the notation, we shall write $\boldsymbol{\omega} \in \mathcal{A}^{\Phi}$ instead of $\boldsymbol{\omega} \in \mathcal{A}^{\Phi}\left(\Omega, \Lambda^{\ell}\right)$, whenever the coordinate forms $\omega_{1}, \ldots, \omega_{m}$ and the Hölder factors $\Phi_{1}, \ldots, \Phi_{m}$ of $\Phi$ play no role in the result.

The following lemma presents the main preliminary estimate relevant to our investigation.

Lemma 2.1. Suppose $0<\lambda<1 /(n+1), 1 / p_{1}+\cdots+1 / p_{m}=1$ and $p_{1}, \ldots, p_{m}>1 /(1-\lambda)$. Let $\boldsymbol{\omega} \in \mathcal{A}^{\mathbf{\Phi}}\left(\Omega, \Lambda^{\ell}\right)$, where $\boldsymbol{\Phi}(t)=\left(t^{p_{1}-\lambda p_{1}}, \ldots, t^{p_{m}-\lambda p_{m}}\right)$. Then for each subcube $\sigma \Omega \subset \Omega, 0<\sigma<1$, we have

$$
f_{\sigma \Omega}|\operatorname{det} \boldsymbol{\omega}|^{1-\lambda} \leq C \prod_{i=1}^{m}\left[\left(f_{\Omega}\left|\omega_{i}\right|^{s_{i}}\right)^{\left(p_{i}-\lambda p_{i}\right) / s_{i}}+\lambda f_{\Omega}\left|\omega_{i}\right|^{p_{i}-\lambda p_{i}}\right]^{1 / p_{i}}
$$

Here the exponents $s_{1}, \ldots, s_{m}$ can be chosen arbitrarily, but must satisfy the conditions: $1 \leq s_{i} \leq(1-\lambda) p_{i}$ and $1 / s_{1}+\cdots+1 / s_{m} \leq 1+1 / n$. The constant $C$ depends only on $\sigma, \lambda, p_{1}, \ldots, p_{m}$ and $s_{1}, \ldots, s_{m}$.

Critical to this lemma is establishing the $L^{p}$-theory of the Hodge decomposition [IM], [IS2]. Actually, inequality (2.10) can be inferred from the proof of Theorem 9.1 given in [IL]. For the convenience of the reader, we shall repeat the relevant arguments of that proof, see Appendix, thus making (2.10) completely established.

One consequence of (2.10) is obtained by letting $\lambda$ go to zero. This gives a generalization of inequality (1.4).

Lemma 2.2. Suppose $\boldsymbol{\Phi}(t)=\left(t^{p_{1}}, \ldots, t^{p_{m}}\right) \log ^{-1}(e+t)$ and $\boldsymbol{\omega} \in \mathcal{A}^{\Phi}\left(\Omega, \Lambda^{\ell}\right)$. Then

$$
f_{\sigma \Omega} \operatorname{det} \boldsymbol{\omega} \leq C\left(f_{\Omega}\left|\omega_{1}\right|^{s_{1}}\right)^{1 / s_{1}} \cdots\left(f_{\Omega}\left|\omega_{m}\right|^{s_{m}}\right)^{1 / s_{m}}
$$

where $1 \leq s_{i}<p_{i}, i=1,2, \ldots, m$, are chosen to satisfy $1 / s_{1}+\cdots+1 / s_{m}=$ $1+1 / n$. 
Here the conditions $\omega_{i} \in \mathcal{L}^{\Phi_{i}}\left(\Omega, \Lambda^{\ell_{i}}\right), \Phi_{i}(t)=t^{p_{i}} \log ^{-1}(e+t)$ for $i=1,2, \ldots$, $m$, are still required though the integrals of $\Phi_{i}\left(\omega_{i}\right)$ do not show up in this inequality. This is in order to ensure that $\lim _{\lambda \rightarrow 0} \lambda \int\left|\omega_{i}\right|^{p_{i}-\lambda p_{i}}=0$, see [Gr1]. Later, we shall see that averaging (2.10) with respect to the parameter $\lambda$ leads to estimates in Orlicz norms, in which the expressions $\|\operatorname{det} \boldsymbol{\omega}\|_{\Psi, \sigma \Omega}$, with $\Psi(t) \prec t$, are controlled by certain Orlicz norms of $\omega_{1}, \ldots, \omega_{m}$, see $(3.17)$.

3. Main results. An easily overlooked fact is that inequality (2.11) is valid with an arbitrary subcube $Q \subset \Omega$ in place of $\Omega$ with the same constant $C=C\left(s_{1}, \ldots, s_{m}, p_{1}, \ldots, p_{m}, \sigma\right)$, thus independent of $Q$. Such "local inequalities" yield a point-wise estimate of the maximal function of the volume form $\omega=$ $\omega_{1} \wedge \cdots \wedge \omega_{m}$ in terms of the maximal functions of $\left|\omega_{1}\right|^{s_{1}}, \ldots,\left|\omega_{m}\right|^{s_{m}}$. As things develop afterwards, we shall need to examine the Hardy-Littlewood maximal operator $M$ in the Orlicz spaces $\mathcal{L}^{\Phi}$ with $\Phi$ not necessarily convex. Suppose $\omega_{i} \in \mathcal{L}^{\Phi_{i}}\left(\Omega, \Lambda^{\ell_{i}}\right)$, where $\Phi_{1}, \ldots, \Phi_{m}$ are Hölder factors of $\Phi$, thus $\omega \in \mathcal{L}^{\Phi}\left(\Omega, \Lambda^{n}\right)$, see (2.9). However, under certain regularity assumptions imposed on $\Phi_{1}, \ldots, \Phi_{m}$, we shall find that also $M \omega \in \mathcal{L}_{\text {loc }}^{\Phi}(\Omega)$. Furthermore, we shall obtain a uniform bound for $M \omega$ on the cube $\sigma \Omega$ :

$$
\|M \omega\|_{\Phi, \sigma \Omega} \leq C\left\|\omega_{1}\right\|_{\Phi_{1}} \cdots\left\|\omega_{m}\right\|_{\Phi_{m}}
$$

for every $0<\sigma<1$, where $C$ depends on $\sigma$. The task is now to deduce from here a higher integrability property of $\omega$. The following proposition deals with this problem.

Proposition 3.1. Suppose the function $\Phi: \mathbb{R}_{+} \rightarrow \mathbb{R}_{+}$is continuously increasing and $\int_{0}^{1} s^{-2} \Phi(s) d s<\infty$. Define an Orlicz function $\Psi: \mathbb{R}_{+} \rightarrow \mathbb{R}_{+}$by

$$
\Psi(t)=\Phi(t)+t \int_{0}^{t} s^{-2} \Phi(s) d s .
$$

Then, for each measurable function $h: \Omega \rightarrow \mathbb{R}_{+}$we have

$$
f_{\Omega} \Psi(h) \leq \Psi\left(f_{\Omega} h\right)+2^{n} f_{\Omega} \Phi(M h) .
$$

Conversely,

$$
f_{\Omega} \Phi(M h) \leq 3^{n} f_{\Omega} \Psi(2 h) .
$$

It is worth pointing out that the term $t \int_{0}^{t} s^{-2} \Phi(s) d s$ represents a convex function. However, $\Psi$ is convex if and only if $\Phi$ is log-convex, the latter being of our interest. 
Remark. Proposition 3.1 extends the well known result of Stein [S2] and refines a result of Bagby and Parsons $[\mathrm{BP}]$. In the sequel, we shall need specific estimates of the maximal functions in Orlicz norms, see inequalities (5.5)-(5.8). As shown in Section 7 our maximal inequalities are sharp, that is, $\Psi$ cannot be replaced by any function of higher growth.

Let $\Phi_{1}, \ldots, \Phi_{m}: \mathbb{R}_{+} \rightarrow \mathbb{R}_{+}$be log-convex functions such that

(3 a) $\Phi_{i} \succeq t^{p_{i}} \log ^{-1}(e+t), i=1,2, \ldots, m$, for some $1<p_{1}, \ldots, p_{m}<\infty, 1 / p_{1}+$ $\cdots+1 / p_{m}=1$.

(3 b) There exist exponents $\alpha_{i} \in\left(1, p_{i}\right)$, with $1 / \alpha_{1}+\cdots+1 / \alpha_{m}<1+1 / n$, for which the functions $t \mapsto t^{-\alpha_{i}} \Phi_{i}(t)$ are increasing, $i=1,2, \ldots, m$.

Next, let $\Phi$ denote the Hölder product of $\Phi_{1}, \ldots, \Phi_{m}$ and let $\Psi$ be defined by (3.1) or, equivalently,

$$
\Phi(t)=\Psi(t)-\int_{0}^{t} \frac{\Psi(s)}{s} d s
$$

Let us notice at once that the condition ( 3 a) yields

$$
\Phi(t) \succeq t \log ^{-1}(e+t)
$$

which, in turn, reveals that

$$
\Psi(t) \succeq t \log \log (e+t)
$$

Under the conditions above for the Orlicz function $\boldsymbol{\Phi}=\left(\Phi_{1}, \ldots, \Phi_{m}\right)$, we may now state one of our main results.

Theorem 1. For each $\boldsymbol{\omega}=\left(\omega_{1}, \ldots, \omega_{m}\right) \in \mathcal{A}^{\mathbf{\Phi}}\left(\Omega, \Lambda^{\ell}\right)$, its volume form $\omega=\omega_{1} \wedge \cdots \wedge \omega_{m}$ belongs to $\mathcal{L}_{\mathrm{loc}}^{\Psi}(\Omega)$. Moreover, for each $0<\sigma<1$ we have

$$
\|\omega\|_{\Psi, \sigma \Omega} \leq C\left\|\omega_{1}\right\|_{\Phi_{1}} \cdots\left\|\omega_{m}\right\|_{\Phi_{m}}
$$

where $C=C\left(\sigma, \Phi_{1}, \ldots \Phi_{m}\right)$.

In fact, the result is sharp. Namely, $\Psi$ cannot be replaced by any function $\Theta$ such that $\lim _{t \rightarrow \infty} \Theta(t) / \Psi(t)=\infty$.

Unfortunately, as noticed by (3.6), our theorem excludes the case $t \preceq \Psi(t) \prec$ $t \log \log (e+t)$. This narrow gap in the scale of integrability of the volume forms will remain unsolved for the remainder of this paper. We put off discussing this problem to Section 6, see also the footnote after Section 6 .

It is easy to see that conditions $(3 \mathrm{a})-(3 \mathrm{~b})$ are not particularly restrictive. Indeed, let $\Phi: \mathbb{R}_{+} \rightarrow \mathbb{R}_{+}$be an arbitrary log-convex function such that 
(3 A) $\Phi(t) \succeq t \log ^{-1}(e+t), \int_{0}^{1} s^{-2} \Phi(s) d s<\infty$.

(3 B) For some $\alpha \in(n /(n+1), 1)$ the function $t \mapsto t^{-\alpha} \Phi(t)$ is increasing.

Having such $\Phi$, we can always define its Hölder factors verifying conditions (3 a) and (3 b), namely,

$$
\Phi_{i}(t)=\frac{1}{p_{i}} \Phi\left(t^{p_{i}}\right), \quad i=1,2, \ldots, m,
$$

where $p_{i}>1 / \alpha$ and $1 / p_{1}+\cdots+1 / p_{m}=1$. Then, one can select numbers $\alpha_{i} \in\left(1, \alpha p_{i}\right)$ to ensure the Sobolev relation $1 / \alpha_{1}+\cdots+1 / \alpha_{m}=1+1 / n$.

Theorem 1 provides a tool for studying the Jacobian function $J=J(x, f)$ of a Sobolev mapping $f=\left(f^{1}, \ldots, f^{n}\right): \Omega \rightarrow \mathbb{R}^{n}$. Indeed, to each $\ell$-tuple $I=\left\{i_{1}, \ldots, i_{\ell}\right\}, 1 \leq i_{1}<\cdots<i_{\ell} \leq n$, there corresponds a closed differential form $\omega^{I}=d f^{i_{1}} \wedge \cdots \wedge d f^{i_{\ell}}$. With this notation we have

$$
\pm J(x, f) d x=\omega^{I_{1}} \wedge \cdots \wedge \omega^{I_{m}}
$$

provided $I_{1}, \ldots, I_{m}$ do not overlap and $I_{1} \cup \cdots \cup I_{m}=\{1, \ldots, n\}$. It is worth noting that the coefficients of $\omega^{I}=d f^{i_{1}} \wedge \cdots \wedge d f^{i_{\ell}}$ are simply the $\ell \times \ell$-minors of the matrix $D f(x) \in G L(n)$, that is,

$$
\frac{\partial\left(f^{i_{1}}, \ldots, f^{i_{\ell}}\right)}{\partial\left(x_{j_{1}}, \ldots, x_{j_{\ell}}\right)}, \quad 1 \leq j_{1}<\cdots<j_{\ell} \leq n .
$$

The advantage of using (3.7) for the differential form of arbitrary degree lies in the fact that the estimate of $\|J\|_{\Psi, \sigma \Omega}$ will depend only on the integrability of certain minors of the differential $D f$. This idea has previously been presented in [IL]. Also, recent applications of differential forms to nonlinear elasticity continue to motivate interest in estimates for differential forms. But we will not develop this point here. Instead, we restrict our attention to the following special case of Theorem 1 .

Theorem 2. Suppose $\Phi$ satisfies (3 A)-(3 B) and $\Psi$ is defined by (3.1). If $f=\left(f^{1}, \ldots, f^{n}\right): \Omega \rightarrow \mathbb{R}^{n}$ is an orientation preserving mapping with $|D f|^{n} \in$ $\mathcal{L}^{\Phi}(\Omega)$, then its Jacobian $J=\operatorname{det} D f$ belongs to $\mathcal{L}_{\text {loc }}^{\Psi}(\Omega)$. Moreover, for each $0<\sigma<1$,

$$
\|J\|_{\Psi, \sigma \Omega} \leq C(n, \sigma, \Phi)\left\||D f|^{n}\right\|_{\Phi}
$$

More precisely,

$$
\|J\|_{\Psi, \sigma \Omega} \leq C\left\|d f^{1}\right\|_{\Theta} \cdots\left\|d f^{n}\right\|_{\Theta}
$$

where $\Theta(t)=\Phi\left(t^{n}\right) / n$. 
We point out that Theorem 1 and Theorem 2 do not involve any assumption about $\Delta_{2}$-regularity of the Orlicz functions. However, condition (3 B) yields $\Phi^{-1}\left(2^{\alpha} t\right) \leq 2 \Phi^{-1}(t)$, which means $\Delta_{2}$-regularity of the inverse function. We also should mention that Theorem 2 was previously established for $\Phi(t)=t \log (e+t)$ in [GI] and then extended to $\Delta_{2}$-regular Orlicz classes in [Mo], see also [Mi2], $[\mathrm{BFS}]$.

However, our main results are concerned with the concave case, that is $\Psi(t) \prec t$ which needs handling with greater care. We should avoid using maximal operators, since the integral averages $f_{Q} \omega$ are no longer available. We succeeded in establishing $\Psi$-summability of the volume forms for those $\Psi: \mathbb{R}_{+} \rightarrow \mathbb{R}_{+}$which can be represented by the following Stieltjes integral

$$
\Psi(t)=\int_{0}^{a}(1-\lambda) t^{1-\lambda} d h(\lambda),
$$

where $h:[0, a] \rightarrow \mathbb{R}_{+}, 0<a<1 /(n+1)$, is an arbitrary non-decreasing function. Thus $\Psi$ is concave and $\Psi(t) \preceq t$. Note that the linear case $\Psi(t)=t$ corresponds to $h(t)=u_{+}(t)$ - the unit step at 0 , that is, $u_{+}(0)=0$ and $u_{+}(t)=1$ for $t>0$. To each such $\Psi$ there corresponds a log-convex function $\Phi: \mathbb{R}_{+} \rightarrow \mathbb{R}_{+}$defined by a formula analogous to (3.4),

$$
\Phi(t)=-\Psi(t)+\int_{0}^{t} \frac{\Psi(s)}{s} d s,
$$

written in terms of $h$ as

$$
\Phi(t)=\int_{0}^{a} \lambda t^{1-\lambda} d h(\lambda) .
$$

In particular, $\Phi(t) \preceq \Psi(t)$. Here is another way of stating (3.12),

$$
\Psi(t)=t \int_{t}^{\infty} s^{-2} \Phi(s) d s-\Phi(t)+t[h(0+)-h(0)] .
$$

For simplicity, we assume, by subtracting the step function $h_{+}=[h(0+)-$ $h(0)] u_{+}$if necessary, that $h$ is continuous at 0 . With this assumption, the relation between $\Phi$ and $\Psi$ is similar to that of (3.1), namely

$$
\Psi(t)=-\Phi(t)+t \int_{t}^{\infty} s^{-2} \Phi(s) d s .
$$

Unfortunately, this excludes the linear case $\Psi(t) \sim t$. On the other hand, such concern would distract us from the presentation of the technique we intend to give. Consequently, the linear case will be discussed in a separate manner.

Let $\Phi_{i}, i=1,2, \ldots, m$, be Hölder factors of $\Phi$ defined by

$$
\Phi_{i}(t)=\frac{1}{p_{i}} \Phi\left(t^{p_{i}}\right)=\frac{1}{p_{i}} \int_{0}^{a} \lambda t^{p_{i}-\lambda p_{i}} d h(\lambda),
$$

where $p_{1}, \ldots, p_{m}$ are Hölder conjugate numbers greater than $1 /(1-a)$. 
Theorem 3. Assume the conditions imposed above on the Orlicz function $\boldsymbol{\Phi}=\left(\Phi_{1}, \ldots, \Phi_{m}\right)$. Let $\boldsymbol{\omega}=\left(\omega_{1}, \ldots, \omega_{m}\right) \in \mathcal{A}^{\Phi}\left(\Omega, \Lambda^{\ell}\right)$. Then its volume form $\omega=\omega_{1} \wedge \cdots \wedge \omega_{m}$ belongs to $\mathcal{L}_{\text {loc }}^{\Psi}(\Omega)$ and for every $0<\sigma<1$ we have

$$
\|\omega\|_{\Psi, \sigma \Omega} \leq C\left\|\omega_{1}\right\|_{\Phi_{1}} \cdots\left\|\omega_{m}\right\|_{\Phi_{m}} .
$$

In particular, for an orientation preserving mapping $f: \Omega \rightarrow \mathbb{R}^{n}$ with $|D f|^{n} \in$ $\mathcal{L}^{\Phi}(\Omega)$, we have

$$
\|\operatorname{det} D f\|_{\Psi, \sigma \Omega} \leq C(n, \sigma, \Phi)\left\||D f|^{n}\right\|_{\Phi} .
$$

Note that $\Phi_{i}$ 's are convex, since $(1-a) p_{i}>1$ for $i=1,2, \ldots, m$, but $\Phi$ is concave. All the functions $\Phi_{1}, \ldots, \Phi_{m}, \Phi$ and $\Psi$ are $\Delta_{2}$-regular. The natural question arises whether a concave function $\Psi$ is equivalent to one given by (3.11). Before answering this question, we recall that our interest is in the case $\Psi \succ \Phi$. This means that $h$ is non-constant near the origin, or equivalently, the support of the measure $d h$ contains the origin. Another way of stating this condition is to say that $\Psi$ grows almost linearly, that is

$$
t \succeq \Psi(t) \succ \Phi(t) \succ t^{1-\varepsilon} \quad \text { for every } \varepsilon>0 .
$$

Under this assumption, $\Phi$ and $\Psi$ are easily checked to be equivalent to the following improper Stieltjes integrals

$$
\Phi(t) \sim \int_{0}^{\infty} \lambda t^{1-\lambda} d h(\lambda) \text { and } \Psi(t) \sim \int_{0}^{\infty} t^{1-\lambda} d h(\lambda),
$$

where $h$ is extended arbitrarily to a bounded and non-decreasing function on $[0, \infty)$. In particular, the upper limit $0<a<1 /(n+1)$ in the integrals $(3.11)$ and (3.13) is not essential for the result stated in Theorem 3. In Section 8, by using the Laplace transform, we completely characterize functions of type (3.18). The criteria for $\Phi$ and $\Psi$ are given in very elementary terms. Some relevant examples are also indicated.

It is of interest to know whether an improvement of integrability of the Jacobian truly takes place. To this effect, we introduce the quotient

$$
L(t)=\frac{\Psi(t)}{\Phi(t)} \geq 1
$$

which measures the degree of the improvement. An easy computation shows that

$$
\Psi(t) \sim t \exp \left[\int_{1}^{t} \frac{d s}{s L(s)}\right] \quad \text { when } \Psi \text { is convex }
$$


and

$$
\Psi(t) \sim t \exp \left[-\int_{1}^{t} \frac{d s}{s L(s)}\right] \quad \text { when } \Psi \text { is concave. }
$$

It is now evident that $L$ cannot grow too fast. Indeed, in order to guarantee $\Psi(t) \succ t$ and $\Psi(t) \prec t$, respectively, we should have

$$
\int_{1}^{\infty} \frac{d s}{s L(s)}=\infty
$$

Roughly speaking, every function $L$, continuously increasing and verifying (3.21), is represented as an improvement quotient $\Psi / \Phi$. Of course, growth conditions imposed on $\Phi$ and $\Psi$ yield other, rather minor, restrictions for $L$.

4. Hölder's inequality. Let $\Phi_{1}, \ldots, \Phi_{m}$ be log-convex and let $\Phi$ be their Hölder product defined by $(2.3)$.

Lemma 4.1 (Young's inequality). For all non-negative numbers $t_{1}, \ldots, t_{m}$ we have

$$
\Phi\left(t_{1} \cdots t_{m}\right) \leq \Phi_{1}\left(t_{1}\right)+\cdots+\Phi_{m}\left(t_{m}\right)
$$

with equality occurring if and only if $t_{1} \Phi_{1}^{\prime}\left(t_{1}\right)=\cdots=t_{m} \Phi_{m}^{\prime}\left(t_{m}\right)$. and

Proof. Denoting $\varphi_{i}(t)=\left(t \Phi_{i}^{\prime}\right)^{-1}$ and $\varphi(t)=\left(t \Phi^{\prime}\right)^{-1}$ we have $\varphi=\varphi_{1} \cdots \varphi_{m}$

$$
\Phi_{i}(t)=\int_{0}^{t} \frac{\varphi_{i}^{-1}(s)}{s} d s \quad \text { and } \quad \Phi(t)=\int_{0}^{t} \frac{\varphi^{-1}(s)}{s} d s .
$$

Hence, by obvious substitutions, we obtain

$$
\Phi_{i}\left(\varphi_{i}(t)\right)=\int_{0}^{t} \frac{s \varphi_{i}{ }^{\prime}(s)}{\varphi_{i}(s)} d s \quad \text { and } \quad \Phi(\varphi(t))=\int_{0}^{t} \frac{s \varphi^{\prime}(s)}{\varphi(s)} d s .
$$

Using the identity $\varphi^{\prime} / \varphi=\varphi_{1}^{\prime} / \varphi_{1}+\cdots \varphi_{m}^{\prime} / \varphi_{m}$ we arrive at the following formula,

$$
\Phi(\varphi(t))=\Phi_{1}\left(\varphi_{1}(t)\right)+\cdots+\Phi_{m}\left(\varphi_{m}(t)\right) .
$$

To prove (4.1), we maximize $\Phi\left(t_{1} \cdots t_{m}\right)$ subject to the constraint

$$
\Phi_{1}\left(t_{1}\right)+\cdots+\Phi_{m}\left(t_{m}\right)=c \quad \text { a given constant. }
$$

With the aid of Lagrange multipliers, we find equations for the extremal tuple $\left(t_{1}, \ldots, t_{m}\right)$. These equations take the form

$$
t_{i} \Phi_{i}^{\prime}\left(t_{i}\right)=t \quad \text { for } i=1,2, \ldots, m,
$$


where $t$ is an unknown positive number. Equivalently, $t_{i}=\varphi_{i}(t)$. Thus, in the extremal case, we have

$$
\begin{aligned}
\Phi_{1}\left(t_{1}\right)+\cdots+\Phi_{m}\left(t_{m}\right) & =\Phi_{1}\left(\varphi_{1}(t)\right)+\cdots+\Phi_{m}\left(\varphi_{m}(t)\right) \\
& =\Phi(\varphi(t))=\Phi\left(\varphi_{1}(t) \cdots \varphi_{m}(t)\right)=\Phi\left(t_{1} \cdots t_{m}\right) .
\end{aligned}
$$

Inequality (4.1) is then straightforward.

Lemma 4.2 (Hölder's inequality). Suppose $\Phi_{i}$ 's are normalized by $\Phi_{i}^{\prime}(1)=$ $1, i=1,2, \ldots, m$. Then

$$
\left\|g_{1} \cdots g_{m}\right\|_{\Phi} \leq\left\|g_{1}\right\|_{\Phi_{1}} \cdots\left\|g_{m}\right\|_{\Phi_{m}}
$$

for all $g_{i} \in \mathcal{L}^{\Phi_{i}}$.

Proof. With the notation $k_{i}=\left\|g_{i}\right\|_{\Phi_{i}}, i=1,2, \ldots, m$, and $k=k_{1} \cdots k_{m}$, we have

$$
f_{\Omega} \Phi_{i}\left(k_{i}{ }^{-1}\left|g_{i}\right|\right) \leq \Phi_{i}(1)
$$

and by Young's inequality

$$
\begin{aligned}
f_{\Omega} \Phi\left(k^{-1}\left|g_{1} \cdots g_{m}\right|\right) & \leq f_{\Omega} \Phi_{1}\left(k_{1}{ }^{-1}\left|g_{1}\right|\right)+\cdots+f_{\Omega} \Phi_{m}\left(k_{m}{ }^{-1}\left|g_{m}\right|\right) \\
& \leq \Phi_{1}(1)+\cdots+\Phi_{m}(1)=\Phi(1) .
\end{aligned}
$$

This latter follows from the identity (4.2) and the normalization, which yields $\varphi_{i}(1)=\varphi(1)=1$, for $i=1,2, \ldots, m$. Hence, by definition of the $\Phi$ functional we get

$$
\left\|g_{1} \cdots g_{m}\right\|_{\Phi} \leq k=\left\|g_{1}\right\|_{\Phi_{1}} \cdots\left\|g_{m}\right\|_{\Phi_{m}}
$$

completing the proof.

5. Maximal inequalities in generalized Orlicz spaces. Let $\Omega$ be a cube in $\mathbb{R}^{n}$ and $0 \leq h \in L^{1}(\Omega)$. The Hardy-Littlewood maximal function of $h$ is defined by

$$
\mathcal{M} h(x)=\sup \left\{f_{Q} h ; x \in Q \subset \Omega\right\},
$$

the supremum being taken over all subcubes of $\Omega$ containing the given point $x \in \Omega$. We begin by recalling two familiar inequalities for the distribution function of $\mathcal{M} h$ :

$$
\lambda(t)=|\{x \in \Omega ; \mathcal{M} h(x)>t\}| .
$$


The first inequality is a consequence of Vitali's covering lemma [S1], [BI]:

$$
\lambda(t) \leq \frac{2 \cdot 3^{n}}{t} \int_{h>t / 2} h(x) d x, \quad \text { for all } t>0
$$

The second one follows from the Calderón-Zygmund decomposition lemma [S1], [BS], and can be viewed as reverse to (5.3):

$$
\lambda(t) \geq \frac{1}{2^{n} t} \int_{h>t} h(x) d x, \quad \text { for } t \geq f_{\Omega} h .
$$

Having disposed of these estimates for the distribution function, we can now prove maximal inequalities (3.2) and (3.3).

Proof of Proposition 3.1. Inequality (3.2) follows quite easily from (5.4). Indeed, with the notation $a=f_{\Omega} h$, we have

$$
\begin{aligned}
\int_{h>a} \Psi(h) & =-\int_{a}^{\infty} \frac{\Psi(t)}{t} d\left(\int_{h>t} h\right) \\
& =\left[-\frac{\Psi(t)}{t} \int_{h>t} h\right]_{a}^{\infty}+\int_{a}^{\infty}\left(\Phi^{\prime}(t) \int_{h>t} h\right) \frac{d t}{t} \\
& \leq \frac{\Psi(a)}{a} \int_{h>a} h+2^{n} \int_{0}^{\infty} \Phi^{\prime}(t) \lambda(t) d t
\end{aligned}
$$

On the other hand, we have an elementary estimate,

$$
\int_{h \leq a} \Psi(h)=\int_{h \leq a} \frac{\Psi(h)}{h} h \leq \frac{\Psi(a)}{a} \int_{h \leq a} h,
$$

because $\Psi(t) / t=\int_{0}^{t} s^{-1} \Phi^{\prime}(s) d s$ is non-decreasing. Summing up, we conclude with

$$
\int_{\Omega} \Psi(h) \leq \frac{\Psi(a)}{a} \int_{\Omega} h+2^{n} \int_{\Omega} \Phi(\mathcal{M} h)
$$

which is the same as (3.2).

To prove (3.3) we can certainly assume that $\Psi(2 h)$ is integrable, since otherwise our inequality holds by trivial means. This assumption yields

$$
\frac{\Psi(t)}{t} \int_{h>t / 2} h \leq \int_{h>t / 2} \frac{\Psi(2 h)}{2 h} h=\int_{h>t / 2} \frac{\Psi(2 h)}{2} \longrightarrow 0
$$


as $t$ goes to $\infty$. We then find that

$$
\begin{aligned}
\frac{1}{2 \cdot 3^{n}} \int_{\Omega} \Phi(\mathcal{M} h) & =\frac{1}{2 \cdot 3^{n}} \int_{0}^{\infty} \Phi^{\prime}(t) \lambda(t) d t \leq \int_{0}^{\infty}\left(\Phi^{\prime}(t) \int_{h>t / 2} h\right) \frac{d t}{t} \\
& \leq\left[-\frac{\Psi(t)}{t} \int_{h>t / 2} h\right]_{0}^{\infty}+\int_{0}^{\infty}\left(\Phi^{\prime}(t) \int_{h>t / 2} h\right) \frac{d t}{t} \\
& =-\int_{0}^{\infty} \frac{\Psi(t)}{t} d\left(\int_{h>t / 2} h\right)=\frac{1}{2} \int_{\Omega} \Psi(2 h)
\end{aligned}
$$

as desired.

Inequalities (3.2) and (3.3) yield information about how the degree of summabilty of $h$ improves, compared to that of $\mathcal{M} h$. Our next objective is to examine maximal inequalities under additional conditions on $\Phi$. Let us first consider the case when there is no improvement, that is

$$
1 \leq \frac{\Psi(t)}{\Phi(t)} \leq q
$$

for some constant $q>1$ and all $t>0$. It follows from (3.1) that

$$
\Phi(t) \geq A t^{p}, \quad A=(p-1) \int_{0}^{1} s^{-2} \Phi(s) d s,
$$

where $p$ is Hölder conjugate to $q$. Conversely, suppose that $\Phi(t)=A(t) t^{p}, p>1$, where $A=A(t)$ is an increasing function. Then

$$
\Phi(t) \leq \Psi(t) \leq q \Phi(t)
$$

as is easy to check. This observation, combined with (3.3), gives the following:

Lemma 5.1. Suppose $A: \mathbb{R}_{+} \rightarrow \mathbb{R}_{+}$is increasing and $\Phi(t)=A(t) t^{p}$ for some $p>1$. Then

$$
\begin{aligned}
\int_{\Omega} \Phi(\mathcal{M} h) & \leq \frac{3^{n} p}{p-1} \int_{\Omega} \Phi(2 h) \leq \int_{\Omega} \Phi\left[2 \cdot\left(\frac{3^{n} p}{p-1}\right)^{1 / p} h\right] \\
& =\int_{\Omega} \Phi\left(C_{p} h\right) \leq k \int_{\Omega} \Phi\left(C_{p} \frac{h}{k}\right)
\end{aligned}
$$

for every $0<k \leq 1$, where $C_{p}^{p}=2^{p} 3^{n} q$. In particular,

$$
\|\mathcal{M} h\|_{\Phi} \leq C_{p}\|h\|_{\Phi} \leq \frac{2 \cdot 3^{n} p}{p-1}\|h\|_{\Phi} .
$$

Concerning inequality (3.2), we have: 
Lemma 5.2. Suppose $\Phi$ is log-convex and $\Psi$ is defined by (3.1). Then

$$
\|h\|_{\Psi} \leq\left(1+2^{n}\right)\|\mathcal{M} h\|_{\Phi} .
$$

Note that in this case $\Psi$ is convex.

Proof. To shorten notation, we introduce the number

$$
k=\frac{\Psi(1)}{\psi(1)+2^{n} \Phi(1)}<1,
$$

thus $1 / k \leq 1+2^{n}$. Inequality (3.2) reads as

$$
f_{\Omega} \Psi\left(\frac{h}{k}\right) \leq \Psi\left(f_{\Omega} \frac{h}{k}\right)+2^{n} f_{\Omega} \Phi\left(\frac{\mathcal{M} h}{k}\right) .
$$

We can certainly assume that $\|\mathcal{M} h\|_{\Phi}=k$, for if not, we replace $h$ by $k h /\|\mathcal{M} h\|_{\Phi}$. Note also that

$$
f_{\Omega} h \leq \inf _{\Omega} \mathcal{M} h \leq\|\mathcal{M} h\|_{\Phi}=k .
$$

Then, by definition of the Luxemburg norm, we obtain

$$
f_{\Omega} \Psi\left(\frac{h}{k}\right) \leq \Psi(1)+2^{n} \Phi(1)=\frac{\Psi(1)}{k} .
$$

As we have mentioned before, $\Psi$ is convex. In particular, $\Psi(h / k) \geq \Psi(h) / k$, for all $0<k \leq 1$. Hence

$$
f_{\Omega} \psi(h) \leq \Psi(1)
$$

which means $\|h\|_{\psi} \leq 1=\|\mathcal{M} h\|_{\Phi} / k \leq\left(1+2^{n}\right)\|\mathcal{M} h\|_{\Phi}$ as desired. lowing,

$$
\Psi^{-1} f_{\Omega} \Psi(h) \leq\left(1+2^{n}\right) \Phi^{-1} f_{\Omega} \Phi(\mathcal{M} h) .
$$

Indeed, since $\Psi$ is convex, its inverse is concave, thus $\Psi^{-1}\left(t_{1}+t_{2}\right) \leq \Psi^{-1}\left(t_{1}\right)+$ $\Psi^{-1}\left(t_{2}\right)$ and $\Psi^{-1}(\lambda t) \leq \lambda \Psi^{-1}(t)$ for $\lambda \geq 1$. Inequality (3.2) implies

$$
\begin{aligned}
\Psi^{-1} f_{\Omega} \Psi(h) & \leq f_{\Omega} h+2^{n} \Psi^{-1} f_{\Omega} \Phi(\mathcal{M} h) \\
& \leq \inf _{\Omega} \mathcal{M} h+2^{n} \Phi^{-1} f_{\Omega} \Phi(\mathcal{M} h) \\
& \leq \Phi^{-1} f_{\Omega} \Phi(\mathcal{M} h)+2^{n} \Phi^{-1} f_{\Omega} \Phi(\mathcal{M} h)
\end{aligned}
$$

as claimed. 
6. Proof of Theorem 1. The main idea is to apply maximal inequalities. According to Lemma 2.2, we have

$$
f_{\sigma Q} \omega \leq C\left(f_{Q}\left|\omega_{1}\right|^{s_{1}}\right)^{1 / s_{1}} \cdots\left(f_{Q}\left|\omega_{m}\right|^{s_{m}}\right)^{1 / s_{m}}
$$

for every subcube $Q \subset \Omega$. Here $s_{1}, \ldots, s_{m}$ are any numbers such that $1 \leq$ $s_{i}<\alpha_{i}, i=1,2, \ldots, m$, see condition $(3 \mathrm{~b})$, and $1 / s_{1}+\cdots+1 / s_{m}=1+1 / n$. Throughout the proof these numbers will be fixed. Thus the constant $C$ in (6.1) is independent of the cube $Q$. For notational simplicity, we denote by $M$ the Hardy-Littlewood maximal operator on the subcube $\sigma \Omega \subset \Omega$. Recall that the script letter $\mathcal{M}$ has been reserved to denote the maximal operator on $\Omega$. Now inequality (6.1) yields

$$
[M \omega](x) \leq C\left[\mathcal{M}\left|\omega_{1}\right|^{s_{1}}\right]^{1 / s_{1}}(x) \cdots\left[\mathcal{M}\left|\omega_{m}\right|^{s_{m}}\right]^{1 / s_{m}}(x)
$$

for all $x \in \sigma \Omega$. We then integrate this over $\sigma \Omega$ and by Young's inequality we obtain

$$
\int_{\sigma \Omega} \Phi\left(\frac{M \omega}{C}\right) \leq \int_{\Omega} \mathcal{F}_{1}\left(\mathcal{M}\left|\omega_{1}\right|^{s_{1}}\right)+\cdots+\int_{\Omega} \mathcal{F}_{m}\left(\mathcal{M}\left|\omega_{m}\right|^{s_{m}}\right)
$$

where $\mathcal{F}_{i}(t)=\Phi_{i}\left(t^{1 / s_{i}}\right), i=1,2, \ldots, m$.

Our next step is to estimate the integrals from the right-hand side by means of the functions $\left|\omega_{i}\right|$. To this effect, we shall make use of Lemma 5.1. Note that the functions $t \mapsto t^{-\alpha_{i} / s_{i}} \mathcal{F}_{i}(t), i=1,2, \ldots, m$, are increasing and $p_{i}=\alpha_{i} / s_{i}>1$. Thus

$$
\int_{\Omega} \mathcal{F}_{i}\left(\mathcal{M}\left|\omega_{i}\right|^{s_{i}}\right) \leq \sigma^{n} \int_{\Omega} \mathcal{F}_{i}\left(C_{p_{i}} \sigma^{-n}\left|\omega_{i}\right|^{s_{i}}\right)=\sigma^{n} \int_{\Omega} \Phi_{i}\left(c_{i}\left|\omega_{i}\right|\right)
$$

where

$$
c_{i}=\left(C_{p_{i}} \sigma^{-n}\right)^{1 / s_{i}}=\left(2 \sigma^{-n}\right)^{1 / s_{i}}\left(\frac{3^{n} \alpha_{i}}{\alpha_{i}-s_{i}}\right)^{1 / \alpha_{i}}, \quad i=1,2, \ldots, m .
$$

Consequently,

$$
f_{\sigma \Omega} \Phi\left(\frac{M \omega}{C}\right) \leq \sum_{i=1}^{m} f_{\Omega} \Phi_{i}\left(c_{i}\left|\omega_{i}\right|\right) .
$$

From Theorem 1, there is no loss of generality in assuming that $\left\|\omega_{i}\right\|_{\Phi_{i}}=1 / c_{i}$ for $i=1,2, \ldots, m$. This gives

$$
f_{\sigma \Omega} \Phi\left(\frac{M \omega}{C}\right) \leq \sum_{i=1}^{m} \Phi_{i}(1)=\Phi(1) .
$$


By the definition of the functional \|\|$_{\Phi}$, we see that

$$
\|M \omega\|_{\Phi, \sigma \Omega} \leq C=C \cdot c_{1} \cdots c_{m}\left\|\omega_{1}\right\|_{\Phi_{1}} \cdots\left\|\omega_{m}\right\|_{\Phi_{m}} .
$$

The final step is to get rid of the maximal operator $M$ on the subcube $\sigma \Omega$. We do this by applying Lemma 5.2 to the operator $M$ in place of the operator $\mathcal{M}$ on $\Omega$. Accordingly,

$$
\|\omega\|_{\Psi, \sigma \Omega} \leq\left(1+2^{n}\right)\|M \omega\|_{\Phi, \sigma \Omega} \leq\left(1+2^{n}\right) C \cdot c_{1} \cdots c_{m}\left\|\omega_{1}\right\|_{\Phi_{1}} \cdots\left\|\omega_{m}\right\|_{\Phi_{m}} .
$$

The proof is complete.

Theorem 2 is now immediate. We simply apply (3.7) to the differential forms $\omega_{i}=d f^{i}$ and Orlicz functions $\Phi_{i}(t)=\Theta(t)=\Phi\left(t^{n}\right) / n, i=1,2, \ldots, m=n$. It remains to observe that

$$
\left\|d f^{i}\right\|_{\Theta}^{n} \leq\|D f\|_{\Theta}^{n}=\left\||D f|^{n}\right\|_{\Phi} .
$$

Remark. We take a moment to discuss possible refinements of the arguments above. Suppose, in addition, that $\Phi_{1}, \ldots, \Phi_{n}$ are convex and $\Delta_{2}$-regular. This latter simply means that $\Phi_{i}(t) \sim t \Phi_{i}^{\prime}(t)$. Consequently,

$$
\Phi^{-1}(t) \sim \Phi_{1}^{-1}(t) \cdots \Phi_{n}^{-1}(t) .
$$

Let $\Theta_{i}, i=1,2, \ldots n$, be $\Delta_{2}$-regular Young functions such that $\Theta_{i} \succ \Phi_{i}$. We assume that $\Theta_{1}, \ldots, \Theta_{n}$ are Sobolev conjugate, that is

$$
\Theta_{1}^{-1}(t) \cdots \Theta_{n}^{-1}(t) \sim t^{1+1 / n} .
$$

Applying the Poincaré-Sobolev Lemma to an orientation preserving mapping $f=$ $\left(f^{1}, \ldots f_{n}\right): \Omega \rightarrow \mathbb{R}^{n}$ with $d f^{i} \in \mathcal{L}^{\Phi_{i}}(\Omega)$, we obtain the following strengthening of (2.11),

$$
f_{\sigma Q} J(x, f) d x \leq C\left\|d f^{1}\right\|_{\Theta_{1}, Q} \cdots\left\|d f^{n}\right\|_{\Theta_{n}, Q}
$$

for each cube $Q \subset \Omega$. As an illustration, consider $\Theta_{1}(t)=\cdots=\Theta_{n}(t)=t^{n^{2} /(n+1)}$ and $\Phi_{1}(t)=\cdots=\Phi_{n}(t)=t^{n}$. This leads to the familiar estimate (1.4), that is

$$
f_{\sigma Q} J(x, f) d x \leq C\left(f_{Q}|D f(x)|^{n^{2} /(n+1)} d x\right)^{(n+1) / n} .
$$


Although the norms $\left\|d f^{i}\right\|_{\Phi_{i}}$ no longer appear in estimate (6.4), they are still required to be finite. For (6.5) to be true, the weakest assumption known up to date is that $|D f|^{n} \in \mathcal{L}^{\Phi}(\Omega)$, where

$$
\Phi(t) \succeq \frac{t}{\log t},
$$

though we conjecture that the same conclusion can be drawn if (6.6) is replaced by an even weaker condition, namely,

$$
\int_{1}^{\infty} s^{-2} \Phi(s) d s=\infty
$$

We also expect that condition (6.7) is sharp. This means that for $\int_{1}^{\infty} s^{-2} \Phi(s) d s<$ $\infty$, there is always an orientation preserving mapping $f=\left(f^{1}, \ldots, f^{n}\right): \Omega \rightarrow \mathbb{R}^{n}$ with $|D f|^{n} \in \mathcal{L}^{\Phi}(\Omega)$ whose Jacobian determinant is not locally integrable. Let us recall that (6.6) yields $\Psi(t) \succeq t \log \log t$. Thus, if the Conjecture was true it would fulfill the gap $t \preceq \Psi(t) \prec t \log \log t$ mentioned in Section 3. ${ }^{1}$

7. An example. In this section we show that the degree of integrability of the volume form as stated in Theorem 1 cannot be improved.

Proposition 7.1. Given an Orlicz function $\Phi: \mathbb{R}_{+} \rightarrow \mathbb{R}_{+}$such that

$$
\int_{0}^{1} s^{-2} \Phi(s) d s<\infty \quad \text { and } \quad \int_{1}^{\infty} s^{-2} \Phi(s) d s=\infty
$$

define $\Psi$ by

$$
\Psi(t)=\Phi(t)+t \int_{0}^{t} s^{-2} \Phi(s) d s
$$

Then, for each $\Theta$ such that $\lim _{t \rightarrow \infty} \Theta(t) / \Psi(t)=\infty$, there exists an orientation preserving mapping $F=\left(f^{1}, \ldots, f^{n}\right): \Omega \rightarrow \mathbb{R}^{n}$ with $|D F|^{n} \in \mathcal{L}^{\Phi}(\Omega)$, whose Jacobian determinant fails to belong to $\mathcal{L}_{\text {loc }}^{\Theta}(\Omega)$.

We give the proof only for the case $n=2$; the other cases can be handled in much the same way.

\footnotetext{
1 Very recently affirmative answers to these questions have been given by C. Li, A. McIntosh, K. Zhang and S. Wu, see [LZ], [LMZ], [Wu]. For another approach see [GIM].
} 
Proof. Given a disk $B=\{x \in \Omega ;|x-a|<r\}$ and $0<\alpha<1$. Consider an orientation preserving mapping

$$
f(x)=a+\frac{r^{\alpha}(x-a)}{|x-a|^{\alpha}} .
$$

It follows easily that

$$
\begin{gathered}
|D f(x)|^{2}=\left(2-2 \alpha+\alpha^{2}\right) \frac{r^{2 \alpha}}{|x-a|^{2 \alpha}} \leq \frac{2 r^{2 \alpha}}{|x-a|^{2 \alpha}} \\
\operatorname{det} D f=(1-\alpha) \frac{r^{2 \alpha}}{|x-a|^{2 \alpha}}
\end{gathered}
$$

Until further notice, we assume that

$$
\int_{1}^{\infty} \frac{\Phi(s)}{s^{\lambda}} d s<\infty, \quad \text { for all } \lambda>2
$$

which, in view of (7.1), implies a similar condition on $\Psi$. Then

$$
\int_{B} \Phi\left(\frac{1}{2}|D f|^{2}\right) \leq \frac{\pi r^{2}}{\alpha} \int_{1}^{\infty} \frac{\Phi(t) d t}{t^{1+1 / \alpha}}
$$

and, for every $k>1$, we have

$$
\int_{B} \Theta\left(\frac{\operatorname{det} D f}{k}\right)=\frac{\pi r^{2}}{\alpha} \int_{1}^{\infty} \frac{\Theta\left(\frac{1-\alpha}{k} t\right)}{t^{1+1 / \alpha}} d t \geq \frac{\pi r^{2}(1-\alpha)}{k^{1 / \alpha} e} \int_{1}^{\infty} \frac{\Theta(t) d t}{t^{1+1 / \alpha}}
$$

Set

$$
\varphi(\alpha)=\frac{1}{\alpha} \int_{1}^{\infty} \frac{\Phi(t) d t}{t^{1+1 / \alpha}}, \quad \psi(\alpha)=(1-\alpha) \int_{1}^{\infty} \frac{\Psi(t) d t}{t^{1+1 / \alpha}}
$$

and

$$
\vartheta(\alpha)=(1-\alpha) \int_{1}^{\infty} \frac{\Theta(t) d t}{t^{1+1 / \alpha}}
$$

for $0<\alpha<1$. Since $\int_{1}^{\infty} s^{-2} \Phi(s) d s=\infty$, we have

$$
\lim _{\alpha \rightarrow 1} \varphi(\alpha)=\infty
$$


Formula (7.1) can be written as $\Phi^{\prime}=t(\Psi / t)^{\prime}$. Hence, integration by parts yields

$$
\begin{aligned}
\psi(\alpha) & =-\alpha \int_{1}^{\infty}\left(t^{1-1 / \alpha}\right)^{\prime}\left(\frac{\Psi}{t}\right) d t \\
& =-\left.\frac{\alpha \Psi(t)}{t^{1 / \alpha}}\right|_{1} ^{\infty}+\alpha \int_{1}^{\infty} t^{1-1 / \alpha}\left(\frac{\Psi}{t}\right)^{\prime} d t \\
& =-\left.\frac{\alpha \Psi(t)}{t^{1 / \alpha}}\right|_{1} ^{\infty}+\frac{1}{\alpha} \int_{1}^{\infty} t^{1-1 / \alpha} \frac{\Phi^{\prime}(t)}{t} d t \\
& =\left.\alpha \frac{\Phi(t)-\Psi(t)}{t^{1 / \alpha}}\right|_{1} ^{\infty}+\int_{1}^{\infty} \frac{\Phi(t)}{t^{1+1 / \alpha}} d t \\
& =\alpha[\Psi(1)-\Phi(1)]+\alpha \varphi(\alpha) \geq \alpha \varphi(\alpha) .
\end{aligned}
$$

Here we have used condition (7.2) for both $\Phi$ and $\Psi$. Next we shall show that

$$
\lim _{\alpha \rightarrow 1} \frac{\vartheta(\alpha)}{\psi(\alpha)}=\infty
$$

Indeed, for each $K>0$ there exists $t_{0}=t_{0}(K)>1$ such that $\Theta(t) \geq K \Psi(t)$ for $t \geq t_{0}$. Splitting the defining integrals for $\psi$ and $\vartheta$ as $\int_{1}^{\infty}=\int_{1}^{t_{0}}+\int_{t_{0}}^{\infty}$, then using $\Psi \geq \Phi$ and (7.3) yields

$$
\liminf _{\alpha \rightarrow 1} \frac{\vartheta(\alpha)}{\psi(\alpha)} \geq K
$$

hence (7.4) follows. Finally, we obtain

$$
\lim _{\alpha \rightarrow 1} \frac{\vartheta(\alpha)}{\varphi(\alpha)}=\infty
$$

Now we shall construct an orientation preserving mapping $F: \Omega \rightarrow \mathbb{R}^{n}$. First, we choose mutually disjoint balls $B_{j}=\left\{x \in \Omega ;\left|x-a_{j}\right|<r_{j}\right\} \subset \Omega, j=1,2, \ldots$, and numbers $0<\alpha_{j}<1$ converging to 1 , such that

$$
\sum_{j=1}^{\infty} \varphi\left(\alpha_{j}\right) r_{j}^{2}<\infty \text { but } \sum_{j=1}^{\infty} \vartheta\left(\alpha_{j}\right) r_{j}^{2}=\infty .
$$

That this is possible follows from the properties of $\varphi$ and $\vartheta$ proven above. We can certainly assume that $\bigcup_{j=1}^{\infty} B_{j}$ is compactly contained in $\Omega$. Put

$$
f_{j}(x)=a_{j}+\frac{r_{j}^{\alpha_{j}}\left(x-a_{j}\right)}{\left|x-a_{j}\right|^{\alpha_{j}}}
$$


for $x \in B_{j}, j=1,2, \ldots$ Then define

$$
F(x)= \begin{cases}f_{j}(x), & \text { if } x \in \bigcup B_{j} \\ x, & \text { if } x \in \Omega \backslash \bigcup B_{j}\end{cases}
$$

We then find that

$$
\int_{B_{j}} \Phi\left(\frac{1}{2}|D F|^{2}\right) \leq \pi r_{j}^{2} \varphi\left(\alpha_{j}\right)
$$

and

$$
\int_{\Omega \backslash \cup B_{j}} \Phi\left(\frac{1}{2}|D F|^{2}\right)=\int_{\Omega \backslash \cup B_{j}} \Phi(1) \leq \Phi(1)|\Omega| .
$$

Therefore,

$$
\int_{\Omega} \Phi\left(\frac{1}{2}|D F|^{2}\right)<\infty
$$

which means that $|D F|^{2} \in \mathcal{L}^{\Phi}(\Omega)$. On the other hand, for each $k \geq 1$ we have

$$
\int_{B_{j}} \Theta\left(\frac{\operatorname{det} D F}{k}\right) \geq \frac{\pi r_{j}^{2}}{e k^{1 / \alpha_{j}}} \vartheta\left(\alpha_{j}\right)
$$

Hence

$$
\int_{\Omega} \Theta\left(\frac{\operatorname{det} D F}{k}\right) \geq \sum_{j=1}^{\infty} \int_{B_{j}} \Theta\left(\frac{\operatorname{det} D F}{k}\right)=\infty
$$

This shows that $\operatorname{det} D F \notin \mathcal{L}_{\text {loc }}^{\Theta}(\Omega)$, as desired.

Note that we did not really have to use the additional hypothesis (7.2); we could have applied our argument to the mappings

$$
f_{j}(x)= \begin{cases}a_{j}+\frac{r_{j}^{\alpha_{j}}\left(x-a_{j}\right)}{\left|x-a_{j}\right|^{\alpha_{j}}}, & \text { if } \varepsilon_{j} \leq\left|x-a_{j}\right| \leq r_{j}, \\ a_{j}+\left(\frac{r_{j}}{\varepsilon_{j}}\right)^{\alpha_{j}}\left(x-a_{j}\right), & \text { if }\left|x-a_{j}\right|<\varepsilon_{j},\end{cases}
$$

where $\varepsilon_{j}$ are chosen sufficiently close to zero, so that the same proof works. The details are left to the reader.

8. Concave case. Here we prove Theorem 3, where the Orlicz functions $\Phi$ and $\Psi$ are given by formulas (3.13) and (3.11), respectively. Notice in advance that these formulas imply that $\Phi$ and $\Psi$ are concave. 
Proof of Theorem 3. The non-trivial case is when $\Psi \succ \Phi$, that is, $0 \in$ supp $d h$. We have already mentioned that $\Phi_{i}$ 's are convex. In view of the assumptions that $p_{i}>1 /(1-a)$ and $0<a<1 /(n+1), 1 / p_{1}+\cdots+1 / p_{m}=1$, it is possible to find numbers $1 \leq s_{i} \leq(1-a) p_{i}, i=1,2, \ldots, m$, such that $1 / s_{1}+\cdots 1 / s_{m}=1+1 / n$. It then follows from (3.15) that $\Phi_{i}(t) \succ t^{\alpha}$ for all $0<\alpha<p_{i}$. In particular, $\omega_{i} \in \bigcap_{\alpha<p_{i}} L^{\alpha}\left(\Omega, \Lambda^{\ell_{i}}\right), i=1,2, \ldots, m$.

Essential to the proof is the estimate $(2.10)$ for $0<\lambda \leq a \leq 1 /(n+1)$, where the constant $C$ does not depend on $\lambda$. By Young's inequality, we deduce from (2.10) that

$$
\begin{aligned}
& f_{\sigma \Omega}\left|\omega_{1} \wedge \cdots \wedge \omega_{m}\right|^{1-\lambda} \\
\leq & C \sum_{i=1}^{m}\left[\frac{1}{p_{i}}\left(f_{\Omega}\left|\omega_{i}\right|^{s_{i}}\right)^{\left(p_{i}-\lambda p_{i}\right) / s_{i}}+\frac{\lambda}{p_{i}} f_{\Omega}\left|\omega_{i}\right|^{p_{i}-\lambda p_{i}}\right] .
\end{aligned}
$$

Integrating against $d h(\lambda)$, in view of the definition of $\Phi_{i}$ and $\Psi$, we find that

$$
f_{\sigma \Omega} \Psi(|\omega|) \leq C \sum_{i=1}^{m}\left[\frac{1}{p_{i}} \Psi\left(\left\|\omega_{i}\right\|_{s_{i}}^{p_{i}}\right)+f_{\Omega} \Phi_{i}\left(\left|\omega_{i}\right|\right)\right] .
$$

Let us recall that $\|g\|_{q}=\left(f_{\Omega}|g|^{q}\right)^{1 / q}$, for every $g$ and every $q \geq 1$. There is no loss of generality in assuming that $\left\|\omega_{i}\right\|_{\Phi_{i}}=1$. This is because (3.16) is not affected if we replace $\omega_{i}$ by a scalar multiple of $\omega_{i}$. Thus

$$
\sum_{i=1}^{m} f_{\Omega} \Phi_{i}\left(\left|\omega_{i}\right|\right)=\sum_{i=1}^{m} \Phi_{i}(1)=\Phi(1)=\int_{0}^{a} \lambda d h(\lambda) .
$$

The only point remaining concerns the $s_{i}$-norms of $\omega_{i}, i=1,2, \ldots, m$, in the right hand side of (8.2). To deal with this, we note that the functions $F_{i}(t)=$ $\Phi_{i}\left(t^{1 / s_{i}}\right)=\Phi\left(t^{p_{i} / s_{i}}\right) / p_{i}$ are convex. By Jensen's inequality we find that

$$
\Phi_{i}\left(\left\|\omega_{i}\right\|_{s_{i}}\right)=F_{i}\left(f_{\Omega}\left|\omega_{i}\right|^{s_{i}}\right) \leq f_{\Omega} F_{i}\left(\left|\omega_{i}\right|^{s_{i}}\right)=f_{\Omega} \Phi_{i}\left(\left|\omega_{i}\right|\right)=\Phi_{i}(1) .
$$

Hence $\left\|\omega_{i}\right\|_{s_{i}} \leq 1$. Combining these inequalities with (8.2), we obtain

$$
f_{\sigma \Omega} \Psi(|\omega|) \leq C[\Psi(1)+\Phi(1)] \leq 2 C \Psi(1) .
$$

For the final conclusion we note that $\Psi\left(t /(2 C)^{1 /(1-a)}\right) \leq \Psi(t) /(2 C)$. Therefore,

$$
f_{\sigma \Omega} \Psi\left(\frac{|\omega|}{(2 C)^{1 /(1-a)}}\right) \leq \Psi(1) .
$$


By definition of the $\Psi$-functional we conclude

$$
\|\omega\|_{\Psi, \sigma \Omega} \leq(2 C)^{1 /(1-a)}=(2 C)^{1 /(1-a)}\left\|\omega_{1}\right\|_{\Phi_{1}} \cdots\left\|\omega_{m}\right\|_{\Phi_{m}},
$$

completing the proof.

The analogue of Theorem 2 in the concave case now reads as follows.

Theorem 4. Let $\Phi, \Psi$ be as in Theorem 3. Suppose $f: \Omega \rightarrow \mathbb{R}^{n}$ is an orientation preserving mapping with $|D f|^{n} \in \mathcal{L}^{\Phi}(\Omega)$. Then $J=\operatorname{det} D f \in$ $\mathcal{L}_{\text {loc }}^{\Psi}(\Omega)$ and

$$
\|J\|_{\Psi, \sigma \Omega} \leq C(n, \sigma)\left\||D f|^{n}\right\|_{\Phi}
$$

More precisely,

$$
\|J\|_{\Psi, \sigma \Omega} \leq C(n, \sigma)\left\|d f^{1}\right\|_{\Theta} \cdots\left\|d f^{n}\right\|_{\Theta},
$$

where $\Theta(t)=\int_{0}^{a} \lambda t^{n-\lambda n} d h(\lambda)$.

Theorems 3 and 4 are far from being conclusive because we still need to know which functions are equivalent to those given by (3.11) and (3.13). Let us first prove formulas (3.18). For $t \geq e^{1 / a}$ we have

$$
0 \leq \int_{a}^{\infty} \lambda t^{1-\lambda} d h(\lambda) \leq a t^{1-a}[h(\infty)-h(a)] \prec \Phi(t) .
$$

Thus

$$
\Phi(t)=\int_{0}^{\infty} \lambda t^{1-\lambda} d h(\lambda)-\int_{a}^{\infty} \lambda t^{1-\lambda} d h(\lambda) \sim \int_{0}^{\infty} \lambda t^{1-\lambda} d h(\lambda) .
$$

Similarly, for $t \geq 1$ we have

$$
0 \leq \int_{a}^{\infty} t^{1-\lambda} d h(\lambda) \leq t^{1-a}[h(\infty)-h(a)] \prec \Psi(t) .
$$

Thus

$$
\begin{aligned}
\Psi(t) & =\int_{0}^{a}(1-\lambda) t^{1-\lambda} d h(\lambda) \sim \int_{0}^{a} t^{1-\lambda} d h(\lambda) \\
& =\int_{0}^{\infty} t^{1-\lambda} d h(\lambda)-\int_{a}^{\infty} t^{1-\lambda} d h(\lambda) \sim \int_{0}^{\infty} t^{1-\lambda} d h(\lambda) .
\end{aligned}
$$

We shall now recall Bernstein's Theorem on the Laplace transform. The best general reference here is the book by D. V. Widder [W]. Accordingly, a function $A=A(s)$ is said to be completely monotonic in $[0, \infty)$ if

$$
(-1)^{k} A^{(k)}(s) \geq 0
$$

for all $0 \leq s<\infty$ and $k=1,2, \ldots$. 
Proposition 8.1 (Bernstein). A necessary and sufficient condition that $A$ should be completely monotonic in $[0, \infty)$ is that

$$
A(s)=\int_{0}^{\infty} e^{-\lambda s} d h(\lambda)
$$

where $h$ is bounded and non-decreasing in $[0, \infty)$.

A useful form of Bernstein's result is obtained by an exponential change of variables

Lemma 8.1. Let $A: \mathbb{R}_{+} \rightarrow \mathbb{R}_{+}$be a bounded decreasing function, completely monotonic in $[0, \infty)$ and such that $1 \succ A(s) \succ e^{-\varepsilon s}$ for every $\varepsilon>0$. Define

$$
\Psi(t)=t A(\log t)
$$

and

$$
\Phi(t)=-t A^{\prime}(\log t) .
$$

Then there is a bounded and non-decreasing function $h:[0, \infty) \rightarrow \mathbb{R}_{+}$which is continuous at zero and such that

$$
\Psi(t) \sim \int_{0}^{a}(1-\lambda) t^{1-\lambda} d h(\lambda) \quad \text { and } \Phi(t) \sim \int_{0}^{a} \lambda t^{1-\lambda} d h(\lambda)
$$

for every $0<a<1$.

Proof. We first observe that the condition $A(s) \succ e^{-\varepsilon s}$ yields $\Psi(t) \succ t^{1-\varepsilon}$. By Bernstein's formula,

$$
A(s)=\int_{0}^{\infty} e^{-\lambda s} d h(\lambda)=\int_{0+}^{\infty} e^{-\lambda s} d h(\lambda)+h(0+)-h(0)
$$

for $s \geq 0$. Here we have $h(0+)=h(0)$, because otherwise $A(s) \sim$ const. $>0$, contradicting the hypothesis that $A(s) \prec 1$. Thus $h$ is continuous at zero and we have

$$
\Psi(t)=t \int_{0}^{\infty} t^{-\lambda} d h(\lambda)=\int_{0}^{\infty} t^{1-\lambda} d h(\lambda)
$$

for $t \geq 1$. From what has already been proved, it follows that

$$
\Psi(t) \sim \int_{0}^{a} t^{1-\lambda} d h(\lambda) .
$$

By a similar argument, we find the formula

$$
\Phi(t) \sim \int_{0}^{a} \lambda t^{1-\lambda} d h(\lambda)
$$

The lemma is completely established. 
Lemma 8.2. If $\chi: \mathbb{R}_{+} \rightarrow \mathbb{R}_{+}$is completely monotonic, then so is the function

$$
A(s)=\exp \left\{-\int_{0}^{s} \chi(t) d t\right\} .
$$

Proof. Write $f(s)=-\int_{0}^{s} \chi(t) d t$. One has to examine the sign of the derivatives of $A$. These are found by using chain and product rules. Indeed,

$$
\begin{aligned}
& A^{\prime}(s)=A(s) f^{\prime}(s) \leq 0, \\
& A^{\prime \prime}(s)=A(s)\left[f^{\prime}(s) f^{\prime}(s)+f^{\prime \prime}(s)\right] \geq 0 .
\end{aligned}
$$

Now, by an induction argument, it is easily seen that

$$
A^{(k)}(s)=A(s) \sum_{I} f^{\left(i_{1}\right)}(s) f^{\left(i_{2}\right)}(s) \cdots f^{\left(i_{\ell}\right)}(s),
$$

where the summation runs over a finite collection (with possible repetitions) of $\ell$-tuples $I=\left(i_{1}, i_{2}, \ldots, i_{\ell}\right)$ of positive integers such that $i_{1}+i_{2}+\cdots+i_{\ell}=k$. Since $\chi$ is completely monotonic, we have $\operatorname{sgn} f^{(i)}=(-1)^{i}, i=1,2, \ldots$. Hence we compute the sign of the monomials under summation

$$
\operatorname{sgn} f^{\left(i_{1}\right)} f^{\left(i_{2}\right)} \cdots f^{\left(i_{\ell}\right)}=(-1)^{k} .
$$

Therefore, $\operatorname{sgn} A^{(k)}=(-1)^{k}$. This shows that $A$ is completely monotonic.

Combining this lemma with Lemma 8.1, we obtain:

Lemma 8.3. Given a completely monotonic function $\chi: \mathbb{R}_{+} \rightarrow \mathbb{R}_{+}$, decreasing to zero and such that $\int_{0}^{\infty} \chi(s) d s=\infty$, define the functions $\Phi$ and $\Psi$ on $[1, \infty)$ by

$$
\begin{gathered}
\Phi(t)=t \chi(\log t) \exp \left[-\int_{0}^{\log t} \chi(s) d s\right], \\
\Psi(t)=t \exp \left[-\int_{0}^{\log t} \chi(s) d s\right] .
\end{gathered}
$$

Then there is a bounded and non-decreasing function $h: \mathbb{R}_{+} \rightarrow \mathbb{R}_{+}$, continuous at zero, such that

$$
\begin{gathered}
\Phi(t)=\int_{0}^{\infty} \lambda t^{1-\lambda} d h(\lambda) \sim \int_{0}^{a} \lambda t^{1-\lambda} d h(\lambda), \\
\Psi(t)=\int_{0}^{\infty} t^{1-\lambda} d h(\lambda) \sim \int_{0}^{a}(1-\lambda) t^{1-\lambda} d h(\lambda),
\end{gathered}
$$

for all $0<a<1$. 
Let us note, for the proof, that the assumptions $\chi(\infty)=0$ and $\int_{0}^{\infty} \chi(s) d s=$ $\infty$ are required only insofar as to ensure the equivalence relations in (8.13) and (8.14). The latter follow from Lemma 8.1, where $A$ is defined by (8.10). Therefore, $e^{-\varepsilon s} \prec A(s) \prec 1$ for every $\varepsilon>0$, as is easy to check. Our last section is dedicated to some relevant examples; see Table 1 and Table 2 . The reader may verify that the function $\chi=\chi(s)$ in the first column of Table 1 is completely monotonic. The functions in the other columns are equivalent to $\Phi, \Psi$ and to the quotient $L=\Psi / \Phi$, respectively.

9. Some conclusions. It is natural to try to formulate what we have done in terms of the improvement quotient $L=\Psi / \Phi$. Let us begin with a brief discussion of the concave case, see formula (3.20). For this, we appeal to Lemma 8.3 where we make the substitution $L(t)=1 / \chi(\log t)$ for $t \geq 1$.

Proposition 9.1. Suppose $L: \mathbb{R}_{+} \rightarrow \mathbb{R}_{+}$increases to infinity slowly enough to satisfy $\int_{1}^{\infty} d s / s L(s)=\infty$. Also assume that the function $\chi(s)=$ $1 / L\left(e^{s}\right)$ is completely monotonic for $s \geq 0$. Define

$$
\Psi(t)=t \exp \left[-\int_{1}^{t} \frac{d s}{s L(s)}\right] \quad \text { and } \quad \Phi(t)=-\Psi(t)+\int_{0}^{t} \frac{\Psi(s)}{s} d s
$$

If $f: \Omega \rightarrow \mathbb{R}^{n}$ is orientation preserving and $|D f|^{n} \in \mathcal{L}^{\Phi}(\Omega)$, then its Jacobian determinant belongs to $\mathcal{L}_{\text {loc }}^{\Psi}(\Omega)$. For each $0<\sigma<1$ we have

$$
\|\operatorname{det} D f\|_{\Psi, \sigma \Omega} \leq C(n, \sigma, L)\left\||D f|^{n}\right\|_{\Phi, \Omega}
$$

We shall now state and prove an analogue of Proposition 9.1 in the convex case, see formula (3.19).

Proposition 9.2. Suppose $L: \mathbb{R}_{+} \rightarrow \mathbb{R}_{+}$is increasing slowly enough to satisfy $L(t) \leq(\log t)(\log \log t)$, for large $t$. Also assume that the function $t \mapsto t^{-a} L(t)$ decreases for some $0<a<1 /(n+1)$. Define

$$
\Psi(t)=t \exp \int_{1}^{t} \frac{d s}{s L(s)} \quad \text { and } \quad \Phi(t)=\Psi(t)-\int_{0}^{t} \frac{\Psi(s)}{s} d s
$$

Then the assertion of Proposition 9.1 remains true.

It is not difficult to verify that in both propositions we have $\Psi \sim L \Phi$. 
Proof. First we show that $\Psi$ is convex. This follows from the elementary inequalities

$$
\left(\frac{t^{1+a} \Psi^{\prime}}{\Psi}-t^{a}\right)^{\prime}=\left(\frac{t^{a}}{L(t)}\right)^{\prime} \geq 0
$$

and hence

$$
\begin{aligned}
\Psi^{\prime \prime} & \geq \frac{(a-1) \Psi}{t^{2}}-\frac{(1+a) \Psi^{\prime}}{t}+\frac{\Psi}{t^{2}}+\frac{\Psi^{\prime 2}}{\Psi} \\
& \geq \frac{(a-1) \Psi}{t^{2}}-\frac{(1+a) \Psi^{\prime}}{t}+\frac{2 \Psi^{\prime}}{t}=(1-a)\left(\frac{\Psi}{t}\right)^{\prime} \geq 0 .
\end{aligned}
$$

Thus $\Psi$ is convex. This also shows, in particular, that $\left(t \Phi^{\prime}\right)^{\prime}=t \Psi^{\prime \prime} \geq 0$. Thus $\Phi$ is log-convex. Next, we show that the function $F(t)=t^{a} \Phi^{\prime}$ is increasing. Indeed,

$$
\begin{aligned}
F^{\prime} & =\left(t^{a} \Psi^{\prime}-t^{a-1} \Psi\right)^{\prime}=(a-1) t^{a-1} \Psi^{\prime}-(a-1) t^{a-2} \Psi+t^{a} \Psi^{\prime \prime} \\
& \geq(a-1) t^{a-1} \Psi^{\prime}-(a-1) t^{a-2} \Psi+(1-a) t^{a}\left(\frac{\Psi}{t}\right)^{\prime}=0 .
\end{aligned}
$$

Now we find that

$$
t^{a-1} \Phi(t)=t^{a-1} \int_{0}^{t} s^{-a} F(s) d s
$$

Hence,

$$
\begin{aligned}
\left(\frac{\Phi(t)}{t^{1-a}}\right)^{\prime} & =(a-1) t^{a-2} \int_{0}^{t} s^{-a} F(s) d s+t^{-1} F(t) \\
& \geq(a-1) t^{a-2} \int_{0}^{t} s^{-a} F(t) d s+t^{-1} F(t)=0 .
\end{aligned}
$$

In other words, the function $t \mapsto \Phi(t) / t^{1-a}$ is increasing, where $1-a=\alpha>$ $n /(n+1)$.

Next, we want to show that $\Phi(t) \geq c t / \log t$ for large $t$. For this, we recall that $L(t) \leq(\log t)(\log \log t)$, which implies $\Phi^{\prime}(t) \geq c / \log t$. Now, for large $t$ we have

$$
\begin{aligned}
\Phi^{\prime}(t) & =\Psi^{\prime}-\frac{\Psi}{t}=t\left(\frac{\Psi}{t}\right)^{\prime}=\frac{1}{L(t)} \exp \int_{1}^{t} \frac{d s}{s L(s)} \\
& \geq \frac{1}{(\log t)(\log \log t)} \exp \left[\int_{e^{e}}^{t} \frac{d s}{s \log s \log \log s}-b\right] \\
& =\frac{1}{(\log t)(\log \log t)} \exp [\log \log \log t-b]=\frac{c}{\log t}
\end{aligned}
$$


Hence,

$$
\Phi(t)=\int_{0}^{t} \Phi^{\prime}(s) d s \geq \int_{t_{0}}^{t} \frac{c d s}{\log s} \geq \frac{c t}{\log t}
$$

as desired. These properties of $\Phi$ make it legitimate to apply Theorem 2, establishing Proposition 9.2.

Table 2 exhibits some triples of functions equivalent to $\Phi, \Psi$ and the improvement quotient $L=\Psi / \Phi$. The table is organized with respect to the degree of the growth of $L$.

10. Appendix. Here we prove Lemma 2.1. As mentioned, the proof can be inferred from the arguments for Theorem 9.1 of [IL]. Recall that $\|g\|_{q}$ stands for $\left(f_{\Omega}|g|^{q}\right)^{1 / q}$.

Let us begin with two quite easy remarks. As $1 / p_{1}+\cdots+1 / p_{m}=1$, at least one of the $p_{i}$ 's is less than $n$ and we suppose $p_{m} \leq n$; thus $s_{m} \leq(1-\lambda) p_{m}<$ $p_{m} \leq n$. Moreover, for $\lambda$ big, say $\lambda \geq 1 / p_{1}$, inequality (2.10) follows immediately from Hadamard's inequality (2.1). Therefore, we can assume $\lambda<1 / p_{1}$.

As, for $i=1,2, \ldots, m$, the $\ell_{i}$-form $\omega_{i}$ is closed, there exists a $\left(\ell_{i}-1\right)$-form $u_{i}$ such that $\omega_{i}=d u_{i}$. Let us fix two cut-off functions $\eta \in C_{0}^{\infty}(\Omega)$ and $\chi \in C_{0}^{\infty}(\Omega)$ such that

$$
\begin{array}{ll}
0 \leq \eta \leq 1, \quad \eta=1 \text { on } \sigma \Omega, & |\nabla \eta| \leq \frac{C(n)}{(1-\sigma) \operatorname{diam} \Omega}, \\
0 \leq \chi \leq 1, \quad \chi=1 \text { on the support of } \eta, & |\nabla \chi| \leq \frac{C(n)}{(1-\sigma) \operatorname{diam} \Omega} .
\end{array}
$$

Next, consider the vectorial form on $\mathbb{R}^{n}$ with compact support

$$
\left(f_{1}, \ldots, f_{m}\right)=\left(\chi\left(u_{1}-u_{1, \Omega}\right), \chi u_{2}, \ldots, \chi u_{m-1}, \eta\left(u_{m}-u_{m, \Omega}\right)\right)
$$

where $u_{1, \Omega}$ and $u_{m, \Omega}$ are suitable closed forms of degree $\left(\ell_{1}-1\right)$ and $\left(\ell_{m}-1\right)$, respectively, defined by (4.25) of [IL]. Since $\eta \neq 0 \Rightarrow \chi=1$, we have the following identity:

$$
\begin{aligned}
& \left|d f_{1}\right|^{-\lambda p_{1}} d f_{1} \wedge \cdots \wedge d f_{m} \\
= & \left|d u_{1}\right|^{-\lambda p_{1}} d u_{1} \wedge \cdots \wedge d u_{m-1} \wedge\left(\eta d u_{m}+d \eta \wedge\left(u_{m}-u_{m, \Omega}\right)\right) .
\end{aligned}
$$

By Theorem 7.1 from [IL], we decompose

$$
\left|d f_{1}\right|^{-\lambda p_{1}} d f_{1}=d \alpha+h,
$$


where both $h$ and $d \alpha$ belong to $\mathcal{L}^{\left(p_{1}-\lambda p_{1}\right) /\left(1-\lambda p_{1}\right)}\left(\mathbb{R}^{n}, \Lambda^{\ell_{1}}\right)$. Moreover,

$$
\|h\|_{\left(p_{1}-\lambda p_{1}\right) /\left(1-\lambda p_{1}\right)} \leq C\left(n, p_{1}\right) \lambda\left\|d f_{1}\right\|_{p_{1}-\lambda p_{1}}^{1-\lambda p_{1}}
$$

By Corollary 4.2 of [IL] (Sobolev-Poincaré inequality),

$$
\left\|d f_{1}\right\|_{p_{1}-\lambda p_{1}} \leq \frac{C\left(n, p_{1}\right)}{1-\sigma}\left\|d u_{1}\right\|_{p_{1}-\lambda p_{1}}
$$

and thus

$$
\|h\|_{\left(p_{1}-\lambda p_{1}\right) /\left(1-\lambda p_{1}\right)} \leq \frac{C\left(n, p_{1}\right)}{1-\sigma} \lambda\left\|d u_{1}\right\|_{p_{1}-\lambda p_{1}}^{1-\lambda p_{1}}
$$

Inserting (10.2) into (10.1) and integrating yields the following formula,

$$
\begin{aligned}
& \int_{\Omega} h \wedge d u_{2} \wedge \cdots \wedge d u_{m-1} \wedge d f_{m} \\
= & \int_{\Omega} h \wedge d f_{2} \wedge \cdots \wedge d f_{m}+\int_{\Omega} d \alpha \wedge d f_{2} \wedge \cdots \wedge d f_{m} \\
= & \int_{\Omega} \eta\left|d u_{1}\right|^{-\lambda p_{1}} d u_{1} \wedge \cdots \wedge d u_{m}+\int_{\Omega}\left|d u_{1}\right|^{-\lambda p_{1}} d u_{1} \wedge \cdots \wedge d u_{m-1} \wedge d \eta\left(u_{m}-u_{m, \Omega}\right),
\end{aligned}
$$

since the integral containing $d \alpha$ vanishes by Stokes Theorem. For this it is important to notice that the forms $d \alpha, d f_{2}, \ldots, d f_{m}$ are integrable with powers $\left(p_{1}-\lambda p_{1}\right) /\left(1-\lambda p_{1}\right), p_{2}-\lambda p_{2}, \ldots, p_{m}-\lambda p_{m}$ and that these powers are Hölder conjugate. Now we use Hölder's inequality to get:

$$
\begin{aligned}
& f_{\Omega} \eta\left|d u_{1}\right|^{-\lambda p_{1}} d u_{1} \wedge \cdots \wedge d u_{m} \\
& \leq\|h\|_{\left(p_{1}-\lambda p_{1}\right) /\left(1-\lambda p_{1}\right)}\left\|d u_{2}\right\|_{p_{2}-\lambda p_{2}} \cdots\left\|d u_{m-1}\right\|_{p_{m-1}-\lambda p_{m-1}}\left\|d f_{m}\right\|_{p_{m}-\lambda p_{m}} \\
& \quad+\sup |d \eta|\left\|\left.d u_{1}\right|^{1-\lambda p_{1}}\right\|_{s_{1}}\left\|d u_{2}\right\|_{s_{2}} \cdots\left\|d u_{m-1}\right\|_{s_{m-1}}\left\|u_{m}-u_{m, \Omega}\right\|_{n s_{m} /\left(n-s_{m}\right)} .
\end{aligned}
$$

The term involving $h$ is estimated by (10.3). By Sobolev-Poincaré inequality

$$
\sup |d \eta|\left\|u_{m}-u_{m, \Omega}\right\|_{n s_{m} /\left(n-s_{m}\right)} \leq \frac{C\left(n, s_{m}\right)}{1-\sigma}\left\|d u_{m}\right\|_{s_{m}}
$$

and

$$
\left\|d f_{m}\right\|_{s_{m}} \leq \frac{C\left(n, s_{m}\right)}{1-\sigma}\left\|d u_{m}\right\|_{s_{m}}
$$


Since $\omega=\operatorname{det} \boldsymbol{\omega}=\omega_{1} \wedge \cdots \wedge \omega_{m}=d u_{1} \wedge \cdots \wedge d u_{m}$ is non-negative, we obtain

$$
\begin{array}{r}
f_{\sigma \Omega}\left|\omega_{1}\right|^{-\lambda p_{1}} \operatorname{det} \boldsymbol{\omega} \leq C \lambda\left\|\omega_{1}\right\|_{p_{1}-\lambda p_{1}}^{1-\lambda p_{1}}\left\|\omega_{2}\right\|_{p_{2}-\lambda p_{2}} \cdots\left\|\omega_{m}\right\|_{p_{m}-\lambda p_{m}} \\
+C\left\|\left.|| \omega_{1}\right|^{1-\lambda p_{1}}\right\|_{s_{1}}\left\|\omega_{2}\right\|_{s_{2}} \cdots\left\|\omega_{m}\right\|_{s_{m}}
\end{array}
$$

where the constant depends on $\sigma$. On the other hand, writing

$$
|\operatorname{det} \boldsymbol{\omega}|^{1-\lambda}=\left|\omega_{1}\right|^{(\lambda-1) \lambda p_{1}}|\operatorname{det} \boldsymbol{\omega}|^{1-\lambda}\left|\omega_{1}\right|^{(1-\lambda) \lambda p_{1}}
$$

and using Hölder's inequality yields

$$
\int_{\sigma \Omega}|\operatorname{det} \boldsymbol{\omega}|^{1-\lambda} \leq\left(\int_{\sigma \Omega}\left|\omega_{1}\right|^{-\lambda p_{1}}|\operatorname{det} \boldsymbol{\omega}|\right)^{1-\lambda}\left(\int_{\sigma \Omega}\left|\omega_{1}\right|^{p_{1}-\lambda p_{1}}\right)^{\lambda},
$$

and thus by (10.4),

$$
\begin{aligned}
f_{\sigma \Omega}|\operatorname{det} \boldsymbol{\omega}|^{1-\lambda} \leq C \lambda\left\|\left|\omega_{1}\right|^{1-\lambda}\right\|_{p_{1}}\left\|\left|\omega_{2}\right|^{1-\lambda}\right\|_{p_{2}} \cdots\left\|\left|\omega_{m}\right|^{1-\lambda}\right\|_{p_{m}} \\
+C\left(f_{\Omega}\left|\omega_{1}\right|^{p_{1}-\lambda p_{1}}\right)^{\lambda}\left\|\left|\omega_{1}\right|^{1-\lambda p_{1}}\right\|_{s_{1}}^{1-\lambda}\left\|\omega_{2}\right\|_{s_{2}}^{1-\lambda} \cdots\left\|\omega_{m}\right\|_{s_{m}}^{1-\lambda} .
\end{aligned}
$$

Moreover, $\left\|\left|\omega_{1}\right|^{1-\lambda p_{1}}\right\|_{s_{1}} \leq\left\|\omega_{1}\right\|_{s_{1}}^{1-\lambda p_{1}}$ and therefore, by Young's inequality with exponents $1 / \lambda p_{1}$ and $1 /\left(1-\lambda p_{1}\right)$, we get

$$
\left(f_{\Omega}\left|\omega_{1}\right|^{p_{1}-\lambda p_{1}}\right)^{\lambda}\left\|\left|\omega_{1}\right|^{1-\lambda p_{1}}\right\|_{s_{1}}^{1-\lambda} \leq \lambda p_{1}\left\|\left|\omega_{1}\right|^{1-\lambda}\right\|_{p_{1}}+\left\|\omega_{1}\right\|_{s_{1}}^{1-\lambda} .
$$

Let us note also that $\left\|\omega_{i}\right\|_{s_{i}}^{1-\lambda} \leq\left\|\left|\omega_{i}\right|^{1-\lambda}\right\|_{p_{i}}$ for $i=1,2, \ldots, m$. Using these remarks we estimate the last term in the right-hand side of (10.5), to obtain

$$
\begin{gathered}
f_{\sigma \Omega}|\operatorname{det} \boldsymbol{\omega}|^{1-\lambda} \leq C \lambda\left\|\left|\omega_{1}\right|^{1-\lambda}\right\|_{p_{1}}\left\|\left|\omega_{2}\right|^{1-\lambda}\right\|_{p_{2}} \cdots\left\|\left|\omega_{m}\right|^{1-\lambda}\right\|_{p_{m}} \\
+C\left\|\omega_{1}\right\|_{s_{1}}^{1-\lambda}\left\|\omega_{2}\right\|_{s_{2}}^{1-\lambda} \cdots\left\|\omega_{m}\right\|_{s_{m}}^{1-\lambda}
\end{gathered}
$$


Finally, note that from this inequality (2.10) follows immediately, since for all $i$,

$$
\begin{array}{r}
\left\{\left(f_{\Omega}\left|\omega_{i}\right|^{s_{i}}\right)^{\left(p_{i}-\lambda p_{i}\right) / s_{i}}+\lambda f_{\Omega}\left|\omega_{i}\right|^{p_{i}-\lambda p_{i}}\right\}^{1 / p_{i}} \\
\geq \max \left\{\lambda^{1 / p_{i}}\left\|\left|\omega_{i}\right|^{1-\lambda}\right\|_{p_{i}},\left\|\omega_{i}\right\|_{s_{i}}^{1-\lambda}\right\} .
\end{array}
$$

\begin{tabular}{|c|c|c|c|}
\hline$\chi=\chi(s)$ & $\Phi=\Phi(t)$ & $\Psi=\Psi(t)$ & $L=L(t)$ \\
\hline$\frac{\alpha}{1+s}, \quad \alpha>0$ & $t \log ^{-1-\alpha} t$ & $t \log ^{-\alpha} t$ & $\log t$ \\
\hline $\begin{array}{l}\frac{\alpha}{(e+s) \log ^{\varepsilon}(e+s)} \\
0<\varepsilon<1, \quad \alpha>0\end{array}$ & $\frac{t e^{-\alpha(\log \log t)^{1-\varepsilon}}}{(\log t)(\log \log t)^{\varepsilon}}$ & $t e^{-\alpha(\log \log t)^{1-\varepsilon}}$ & $(\log t)(\log \log t)^{\varepsilon}$ \\
\hline $\begin{array}{l}\frac{\alpha}{(e+s) \log (e+s)}, \\
\alpha>0\end{array}$ & $\frac{t}{(\log t)(\log \log t)^{1+\alpha}}$ & $\frac{t}{(\log \log t)^{\alpha}}$ & $(\log t)(\log \log t)$ \\
\hline $\begin{array}{l}\frac{1}{\ell_{0}(s) \ell_{1}(s) \cdots \ell_{k-1}(s)} \\
\ell_{0}(s)=s \\
\ell_{k}=\log \left(e+\ell_{k-1}\right)\end{array}$ & $\frac{t}{\ell_{1}(t) \cdots \ell_{k-1}(t) \ell_{k}^{2}(t)}$ & $\frac{t}{\ell_{k}(t)}$ & $\ell_{1}(t) \cdots \ell_{k}(t)$ \\
\hline $\begin{array}{l}\alpha e^{-\beta \log ^{\varepsilon}(1+s)} \\
0<\varepsilon<1, \quad \alpha, \beta>0\end{array}$ & $\ldots$ & $\cdots$ & $e^{\beta(\log \log t)^{\beta}}$ \\
\hline $\begin{array}{l}\frac{\alpha}{(1+s)^{1-\varepsilon}} \\
0<\varepsilon<1, \quad \alpha>0\end{array}$ & $\frac{t}{(\log t)^{1-\varepsilon} e^{\alpha \log ^{\varepsilon} t}}$ & $\frac{t}{e^{\alpha \log ^{\varepsilon} t}}$ & $(\log t)^{1-\varepsilon}$ \\
\hline
\end{tabular}

TABLE 1 
TABLE 2

\begin{tabular}{|c|c|c|}
\hline$\Psi=\Psi(t)$ & $\Phi=\Phi(t)$ & $L=L(t)$ \\
\hline$t \log \log t$ & $t / \log t$ & $(\log t) \log \log t$ \\
\hline$t(\log \log t)^{\gamma}, \quad \gamma \geq 1$ & $\frac{\gamma t(\log \log t)^{\gamma-1}}{\log t}$ & $\frac{1}{\gamma}(\log t) \log \log t$ \\
\hline$t(\log t)^{\alpha}, \quad \alpha>0$ & $\frac{\alpha t(\log t)^{\alpha-1}}{\operatorname{loxp}\left[\beta(\log t)^{\varepsilon}\right],}$ & $\frac{1}{\alpha} \log t$ \\
\hline$\beta>0, \quad 0<\varepsilon<1$ & $\frac{\varepsilon \beta t \exp \left[\beta(\log t)^{\varepsilon}\right]}{\log { }^{1-\varepsilon} t}$ & $\frac{1}{\varepsilon \beta} \log { }^{1-\varepsilon} t$ \\
\hline$t \exp \left[\frac{\beta \log t}{\log \log t}\right]$, & $\frac{\beta t \exp \left[\frac{\beta \log t}{\log \log t}\right]}{\log \log t}$ & $\frac{1}{\beta} \log \log t$ \\
\hline$\beta>0$ & $\frac{\beta t \exp \left[\frac{\beta \log t}{\ell_{k}(t)}\right]}{\ell_{k}}$ & $\frac{1}{\beta} \ell_{k}(t)$ \\
\hline $\exp \left[\frac{\beta \log t}{\ell_{k}(t)}\right], \quad \beta>0$, \\
$k=2,3, \ldots$
\end{tabular}

\section{REFERENCES}

[B] J. M. BALL, Convexity conditions and existence theorems in nonlinear elasticity, Arch. Rational Mech. Anal. 63, 337-403.

[BFS] H. Brezis, N. Fusco \& C. Sbordone, Integrability for the Jacobian of orientation preserving mappings, J. Funct. Anal. 115 (1993), 425-431.

[BI] B. BoJARSKI $\&$ T. IWANIEC, Analytical foundations of the theory of quasiconformal mappings in $\mathbb{R}^{n}$, Ann. Acad. Sci. Fenn. Ser. A I Math. (1983), 257-324.

[BM] J. M. BALL \& F. MURAT, $W^{1, p}$-quasi-convexity and variational problems for multiple integrals, J. Funct. Anal. 58 (1984), 225-253.

[BP] R. BAgBy 63 D. PARSON, Orlicz Spaces and rearranged maximal funtions, Math. 
Nachr. 132 (1987), 15-27.

[BS] C. Bennet 8 R. Sharpley, Interpolation of Operators, Academic Press, New York, 1988.

[ClmS] R. R. Coifman, P. L. Lions, Y. Meyer \& S. Semmes, Compacité par compensation et éspaces de Hardy, C. R. Acad. Sci. Paris Sér I Math. 309 (1989), 945-949.

[DMa] B. Dacorogna \& P. MARCELlini, Semicontinuité pour des integrandes polyconvexes sans continuité des determinants, C. R. Acad. Sci. Paris Sér. I Math. 311 (1990), 393-396.

[DMu] B. Dacorogna \& F. Murat, On the optimality of certain Sobolev exponents for the weak continuity of determinants, (Preprint) (1995).

[Do] A. Dolcini, A Div-Curl result for the Jacobian, Rend. Accad. Sci. Fis. Mat. Napoli LX (1993), 113-120.

[G] F. W. GEHRING, The $L^{p}$-integrability of the partial derivatives of a quasiconformal mapping, Acta Math. 130 (1973), 265-277.

[Gr1] L. GReCO, A remark on the equality $\operatorname{det} D f=\operatorname{Det} D f$, Differential Integral Equations 6 (1993), 1089-1100.

[Gr2] _ Su alcune Proprietà dei Determinanti Jacobiani, Ph.D Thesis, University of Naples, 1993.

[GI] L. Greco \& T IWANIEC, New inequalities for the Jacobian, Ann. Inst. H. Poincaré, 11 (1994), 17-35.

[GIM] L. Greco, T. IwanieC $\mathscr{G}$ M. Milman, in preparation.

[I1] T. IWANIEC, $L^{p}$-Theory of Quasiregular Mappings, Lecture Notes in Math., 1508, Springer-Verlag (1992).

[I2],$p$-Harmonic tensors and quasiregular mappings, Ann. of Math. 136 (1992), $589-624$.

[I3] Integrability theory of the Jacobians, Preprint of the University of Bonn, Lipschitz Lectures, June 15-July 16, 1995.

[IL] T. IWANIEC $\&$ A. Lutoborski, Integral estimates for null Lagrangians, (Preprint).

[IM] T. IWANIEC $\&$ G. MARTIN, Quasiregular mappings in even dimension, Acta Math. 170 (1993), 29-81.

[IS1] T. IWANIEC \& C. SBordone, On the integrability of the Jacobian under minimal hypothesis, Arch. Rational Mech. Anal. 119 (1992), 129-143.

[IS2] — Weak minima of variational integrals, J. Reine Angew. Math. 454 (1994), $143-161$.

[KR] M. A. Krasnosel'skit \& YA. B. Rutickit, Convex Functions and Orlicz Spaces, Noordhoff, Groningen, 1961.

[L] P. L. Lions, Jacobians and Hardy spaces, Ricerche Mat. Suppl. 40 (1991).

[LMWZ] C. Li, A. McIntosh, Z. Wu \& K. ZhAng, Compensated compactness, paracompactness, and Hardy spaces, J. Funct. Anal. (to appear).

[LMZ] C. Li, A. McIntosh \& K. Zhang, Higher Integrability and Reverse Hölder Inequalities, (Preprint).

[LZ] C. Li \& K. ZHANG, Higher Integrability of certain Bilinear Forms on Orlicz Spaces, (Preprint).

[M] S. MüLLER, Higher integrability of determinants and weak convergence in $L^{1}, \mathrm{~J}$. Reine Angew Math. 412 (1990), 20-34. 
[Mi1] M. Milman, A Commutator Theorem with Applications, Institute for Advanced Study, 1992, (Preprint).

[Mi2] - Inequalities for the Jacobian: Interpolation Techniques, $2^{\text {nd }}$ Latin American Analysis Conference, Bogota, 1992.

[Mi3] _ Integrability of the Jacobian of orientation preserving maps: Interpolation methods, (Preprint).

[Mo] G. Moscariello, On the integrability of the Jacobian in Orlicz Spaces, Math. Japonica 40 (1992), 323-329.

[R] YU. G. RECHETNYAK, On the stability of conformal mappings in multidimensional spaces, Siberian Math. J. 8 (1967), 65-85.

[RR] M. M. RAO E Z. D. REN, Theory of Orlicz Spaces, Marcel Dekker, CITY?, 1991.

[RW] R. Rochberg \& G. WeIss, Derivatives of Analytic families of Banach spaces, Ann. of Math. 118 (1983), 315-347.

[S1] E. M. SteIn, Singular Integrals and Differentiability Properties of Functions, Princeton University Press, Princeton, 1970.

[S2] - Note on the class L $\log L$, Studia Math. 32 (1969), 305-310.

[T] L. TARTAR, Hardy's spaces and applications, CMU Seminar, University of Pittsburgh.

[V] J. VÄISÄL ̈̈, Lectures on n-dimensional quasiconformal mappings, Lecture Notes in Math., 229, Springer Verlag (1971).

[W] D. V. WIDDER, The Laplace Transform, Princeton University Press, Princeton, 1946.

[Wu $\mathrm{S} . \mathrm{Wu}$, On the higher integrability of nonnegative Jacobians, (Preprint).

[Z] Y. ZHOU, An $L^{p}$-Theory of the compensated compactness, Proc. Roy. Soc. Edinburg Sect. A 112 (1992), 177-189.

The first and third authors were supported in part by M.U.R.S.T. (1990). The second author was supported in part by NSF Grant DMS-9401104 and Istituto per le Applicazioni della Matematica, CNR, Napoli.

\section{LUIGI GRECO}

Dipartimento di Matematica e Applicazioni

"R. Caccioppoli", Università

Via Cintia, Complesso Monte S.Angelo

80126 Napoli, Italy

Gioconda Moscariello

Dipartimento di Matematica e Applicazioni

"R. Caccioppoli", Università

Via Cintia, Complesso Monte S.Angelo

80126 Napoli, Italy
TADEusz IWANIEC

Department of Mathematics

Syracuse University

Syracuse, New York 13244

Received: November 29, 1993; revised: March 24, 1995. 